\title{
Planetary Gearbox Fault Diagnosis via Torsional Vibration Signal Analysis in Resonance Region
}

\author{
Kangqiang Li, ${ }^{1}$ Zhipeng Feng, ${ }^{1}$ and Xihui Liang ${ }^{2}$ \\ ${ }^{1}$ School of Mechanical Engineering, University of Science and Technology Beijing, Beijing 100083, China \\ ${ }^{2}$ Department of Mechanical Engineering, University of Alberta, Edmonton, AB, Canada T6G 2G8 \\ Correspondence should be addressed to Zhipeng Feng; fengzp@ustb.edu.cn
}

Received 20 August 2017; Accepted 29 October 2017; Published 21 November 2017

Academic Editor: Rafał Burdzik

Copyright (c) 2017 Kangqiang Li et al. This is an open access article distributed under the Creative Commons Attribution License, which permits unrestricted use, distribution, and reproduction in any medium, provided the original work is properly cited.

\begin{abstract}
Planetary gearbox torsional vibration signals are free from the extra amplitude modulation effect due to time-varying transmission paths and have simpler frequency structure than translational ones. Gear faults result in modulation on the torsional resonance vibration and are manifested by the modulation feature. These merits are exploited for planetary gearbox fault diagnosis in this paper. Gear fault induced torsional vibrations in resonance region are modelled as amplitude modulation and frequency modulation (AM-FM) processes, the explicit equation of Fourier spectrum is derived, and the sideband characteristics are summarized. To avoid complex sideband analysis, amplitude and frequency demodulation analysis methods are exploited. The equations of amplitude and frequency demodulated spectra are derived in closed form, and their frequency structures are revealed. For fault diagnosis based on above theoretical derivations, a resonance frequency identification approach is proposed through time-frequency analysis of torsional vibrations during variable speed processes, according to the independence nature of resonance frequency on running conditions. The theoretical derivations and proposed approach are illustrated by numerical simulated signal analysis and are further validated through dynamics modelling and lab experimental tests. Localized faults on the sun, planet, and ring gears are successfully diagnosed.
\end{abstract}

\section{Introduction}

Planetary gearboxes are widely used in various types of machines, such as wind turbines, helicopters, and trucks. Once fault occurs in a planetary gearbox, it may lead to reduction in transmission efficiency and even breakdown of the entire drive train. Hence, fault diagnosis has great significance to maintain reliable operation of planetary gearboxes $[1,2]$.

To date, researchers have made many important contributions to planetary gearbox fault diagnosis, but they mainly focus on translational vibration signals. To name a few, for example, McFadden [3, 4] and Samuel and Pines [5], respectively, proposed a vibration separation method of planet and sun gears by generalizing the time domain averaging method. Liang et al. [6] developed a signal decomposition technique to extract the vibration signal corresponding to one tooth of a planet gear for planet gear tooth crack detection. Barszcz and Randall [7] applied spectral kurtosis to gear fault detection, and they diagnosed the ring gear tooth crack in a wind turbine planetary gearbox. Lei et al. [8] proposed an adaptive stochastic resonance method to suppress noise interference and enhance fault signature and applied it to weak fault feature extraction of planetary gearboxes. Yoon et al. [9] developed spectral averaging via enveloping and Welch's spectral averaging for planetary gearbox fault diagnosis. These works have enriched our understanding on planetary gearbox fault diagnosis.

Translational vibration signals are subject to extra amplitude modulation (AM) effect caused by the planet carrier rotation, and they exhibit intricate spectral structures. Translational vibration signals are usually measured by sensors fixed on gearbox casing or bearing housing. The distance from sun-planet and planet-ring gear meshing locations to the sensor varies with the planet carrier rotation. This results in a time variant vibration transfer path and thereby an extra AM effect on translational vibrations. McFadden and Smith 
[10], McNames [11], and Mosher [12] found that the translational vibration signal spectrum of planetary gearboxes is typically asymmetric due to the planet carrier rotation. The spectral complexity leads to difficulty in planetary gearbox fault diagnosis via translational vibration signal analysis.

Torsional vibration signals are free from the extra AM effect due to the planet carrier rotation and show simpler spectral structures than translational ones. Torsional vibration sensors are usually connected to the input or output shaft of a gearbox, and the distance from sun-planet and planetring gear meshing locations to the sensor is isotropic circumferentially. As such, the torsional vibration transfer paths are time invariant, even though the sun-planet and planetring gear meshing locations vary during gearbox running. Inspired by this property, Feng and Zuo [13] exploited the merit of torsional vibrations to avoid the intricate spectral structure existing with translational vibrations and extracted the sun, planet, and ring gear fault feature around gear meshing frequency or its harmonics.

Gear fault information is not only carried by gear meshing vibrations, but also conveyed by resonances [14, 15]. Localized defects on a gear tooth surface will generate sudden changes in torque as it meshes with mating gears. These sudden changes will further excite torsional resonances. Torsional resonances vanish due to damping effect, but they arise repetitively at the gear fault frequency during gearbox running. In this sense, the resonance region contains gear fault information. However, to our best knowledge, research on planetary gearbox fault diagnosis via torsional vibration signal analysis, particularly in the resonance region, has been very limited.

In this paper, we propose to extract gear fault torsional vibration signature in resonance region. To implement this idea, we firstly identify the torsional resonance frequency via time-frequency analysis of torsional vibration signals during variable speed processes. This resonance frequency identification method overcomes the difficulty in exciting torsional resonances via traditional hammer impact technique. Then, we extract gear fault features around the resonance frequency via sideband analysis in Fourier spectrum, and amplitude and frequency demodulation analysis. In summary, this proposed methodology does not only offer an alternative solution to avoid the complexity issue existing with translational vibrations due to extra amplitude modulation effect by the time-varying vibration propagation paths, but also provides a new insight into gear fault induced torsional vibration signatures.

Hereafter, this paper is organized as follows. Section 2 derives the explicit equations of Fourier spectrum, envelope spectrum, and the Fourier spectrum of instantaneous frequency in the resonance frequency region, respectively. Section 3 introduces the resonance frequency identification approach and the sensitive component separation method and summarizes the analysis procedure as well. Section 4 illustrates the torsional resonance vibration characteristics via a numerically simulated signal analysis. Sections 5 and 6 validate the proposed method via dynamics modelling data of a planetary gear set and lab experimental signals of a planetary gearbox. Section 7 draws conclusions.

\section{Gear Fault Characteristics in Torsional Resonance Region}

In this section, we extract gear fault features in the torsional resonance frequency region, instead of around gear meshing frequency and its harmonics that are usually focused in conventional methods. Therefore we derive explicit equations of Fourier spectrum, envelope spectrum, and Fourier spectrum of instantaneous frequency, for better understanding their spectral structure.

2.1. Torsional Vibration Signal Model in Resonance Region. Suppose we have a localized fault on the tooth surface of sun, planet, or ring gear. Under constant speed operation, as the fault area strikes mating gear teeth, sudden changes in torque will be generated. Consequently, the repeating sudden torque changes excite the torsional resonance of gear-shaft system periodically. Such resonance vanishes rapidly due to damping before the next resonance comes, resulting in the AM phenomenon. Meanwhile, in one repeating cycle, the resonance exists in the early portion and the instantaneous frequency equals approximately the resonance frequency, while in the later portion, the resonance vanishes due to damping and the instantaneous frequency becomes 0 . This means the instantaneous frequency changes periodically, resulting in frequency modulation (FM). Therefore, the fault exciting torsional vibration signals around resonance frequency can be modelled as an amplitude modulation and frequency modulation (AM-FM) process, with the carrier frequency equal to resonance frequencies and the modulating frequency equal to the gear fault frequency harmonics.

Without loss of generality, we focus on one resonance frequency $f_{n}$ and the fundamental gear fault frequency $f_{g}$ only. Then, the torsional vibration signal model in the resonance region can be simplified as

$$
\begin{aligned}
x(t)= & {\left[1+A \cos \left(2 \pi f_{g} t+\phi\right)\right] } \\
& \cdot \cos \left[2 \pi f_{n} t+B \sin \left(2 \pi f_{\mathrm{g}} t+\varphi\right)+\theta\right],
\end{aligned}
$$

where $A>0$ and $B>0$ are the AM and FM magnitude, respectively, and $\phi, \varphi$, and $\theta$ are the initial phases.

2.2. Fourier Spectrum. According to the property of Bessel functions [17],

$$
\exp [j z \sin (\theta)]=\sum_{m=-\infty}^{\infty} J_{m}(z) \exp (j m \theta),
$$

where $J_{m}(z)$ is the first class of Bessel function with integer order $m$ and argument $z$ and the identities of trigonometric functions. Then, (1) can be rewritten as

$$
\begin{gathered}
x(t)=\left[1+A \cos \left(2 \pi f_{\mathrm{g}} t+\phi\right)\right] \times \sum_{m=-\infty}^{\infty} J_{m}(B) \\
\cdot \cos \left[2 \pi\left(f_{n}+m f_{g}\right) t+m \varphi+\theta\right]=\sum_{m=-\infty}^{\infty} J_{m}(B)
\end{gathered}
$$




$$
\begin{aligned}
& \cdot \cos \left[2 \pi\left(f_{n}+m f_{g}\right) t+m \varphi+\theta\right]+\frac{A}{2} \sum_{m=-\infty}^{\infty} J_{m}(B) \\
& \cdot \cos \left\{2 \pi\left[f_{n}+(m+1) f_{g}\right] t+m \varphi+\theta+\phi\right\}+\frac{A}{2} \\
& \cdot \sum_{m=-\infty}^{\infty} J_{m}(B) \\
& \cdot \cos \left\{2 \pi\left[f_{n}+(m-1) f_{g}\right] t+m \varphi+\theta-\phi\right\} .
\end{aligned}
$$

Applying Fourier transform to (3) yields the Fourier spectrum

$$
\begin{aligned}
& X(f)=\sum_{m=-\infty}^{\infty} J_{m}(B) \delta\left[f-\left(f_{n}+m f_{g}\right)\right] \\
& \cdot \exp [j(m \varphi+\theta)]+\frac{A}{2} \sum_{m=-\infty}^{\infty} J_{m}(B) \\
& \cdot \delta\left\{f-\left[f_{n}+(m+1) f_{g}\right]\right\} \exp [j(m \varphi+\theta+\phi)] \\
& +\frac{A}{2} \sum_{m=-\infty}^{\infty} J_{m}(B) \delta\left\{f-\left[f_{n}+(m-1) f_{g}\right]\right\} \\
& \cdot \exp [j(m \varphi+\theta-\phi)],
\end{aligned}
$$

where $\delta(\cdot)$ denotes the Dirac delta function.

According to (4), peaks appear in the Fourier spectrum at frequency locations $f_{n} \pm m f_{g}$. They form sidebands around the resonance frequency $f_{n}$, with a sideband spacing equal to the gear fault frequency $f_{g}$. Based on such characteristics, we can diagnose gear fault according to the presence of sidebands or changes in their magnitudes, and particularly the associated sideband spacing, in the resonance frequency region.

2.3. Envelope Spectrum. For the signal model (1), the AM part

$$
e(t)=1+A \cos \left(2 \pi f_{g} t+\phi\right)
$$

contains gear fault information, because its modulating frequency equals the gear fault frequency $f_{g}$. This motivates us to reveal gear fault signature via amplitude demodulation analysis.

Applying Fourier transform to (5) yields the envelope spectrum

$$
E(f)=\delta(f)+A \delta\left(f-f_{g}\right) \exp (j \phi)
$$

According to (6), in the envelope spectrum, peak appears at the gear fault frequency $f_{g}$ only. If we consider higher order harmonics of amplitude modulating frequencies, peaks will also appear at the gear fault frequency harmonics $k f_{g}$. Therefore, we can detect gear fault according to the existence of gear fault frequency harmonics $k f_{g}$ or increase in their magnitudes in the envelope spectrum.
2.4. Fourier Spectrum of Instantaneous Frequency. According to the signal model (1), the FM part also contains gear fault information, since its modulating frequency is the gear fault frequency. Hence, we can detect gear fault via frequency demodulation analysis. is

For the signal model, given in (1), its instantaneous phase

$$
\alpha(t)=2 \pi f_{n} t+B \sin \left(2 \pi f_{g} t+\varphi\right)+\theta .
$$

The instantaneous frequency can be derived as a derivative of the instantaneous phase $\alpha(t)$ with respect to time $t$ :

$$
f(t)=\frac{1}{2 \pi} \frac{d \alpha(t)}{d t}=f_{n}+B f_{g} \cos \left(2 \pi f_{g} t+\varphi\right) .
$$

Applying Fourier transform to (8) yields the Fourier spectrum of instantaneous frequency

$$
F(f)=f_{n} \delta(f)+B f_{g} \delta\left(f-f_{g}\right) \exp (j \varphi) .
$$

Equation (9) implies that peaks appear at the gear fault frequency $f_{g}$ in addition to 0 , in the Fourier spectrum of instantaneous frequency. If we consider higher harmonics of the frequency modulating frequency, peaks will also exist at the gear fault frequency harmonics $k f_{g}$. Based on such features, we can detect gear fault according to the frequency locations of peaks present in the Fourier spectrum of instantaneous frequency.

\section{Analysis Procedure}

Figure 1 shows the flowchart of our proposed analysis method. Firstly, we identify the torsional resonance frequency via time-frequency analysis of torsional vibration signals during variable speed processes (to be explained in Section 3.1). Then, we extract gear fault symptoms by sideband analysis around the resonance frequency in Fourier spectrum. Next, we separate the resonance component via bandpass filtering around the resonance frequency and discern gear fault signature in its envelope spectrum. We further decompose the filtered resonance component into monocomponents via ensemble empirical mode decomposition (EEMD), choose a sensitive monocomponent (to be introduced in Section 3.2), and pinpoint gear fault information from the Fourier spectrum of its instantaneous frequency. Finally, we diagnose gear fault by combining the findings from sideband analysis in Fourier spectrum, and amplitude and frequency demodulation analyses.

3.1. Torsional Resonance Frequency Identification. To extract gear fault signatures around torsional resonance frequency, it is necessary to identify the resonance frequency first. Hammer impact technique is commonly used in translational resonance identification. However, it is difficult to apply an impact to a gear-shaft torsional vibration system. To overcome this difficulty, we exploit the independence nature of resonance frequency on running conditions, particularly the running speed, and propose a resonance frequency identification approach via time-frequency analysis. 


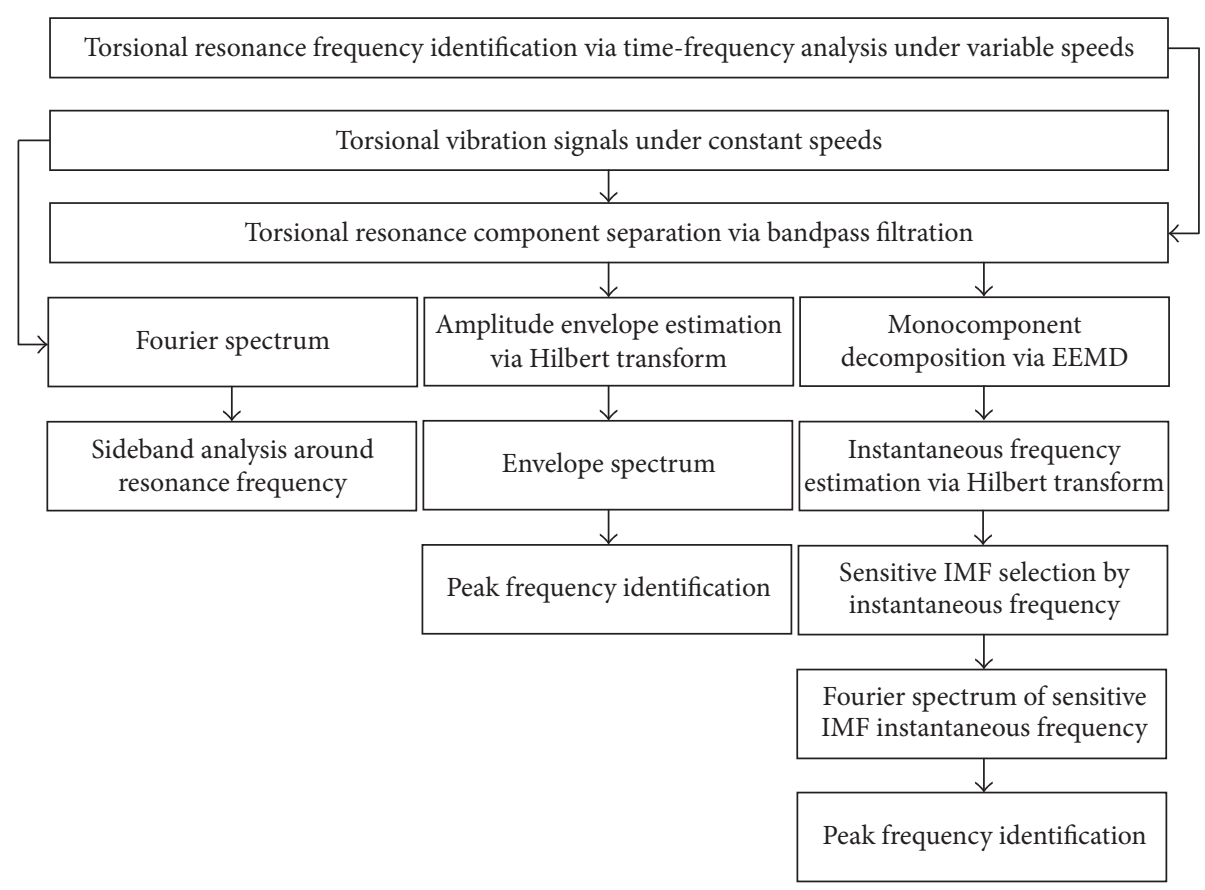

Figure 1: Analysis flowchart.

Gear meshing and resonance vibrations are major components of gearbox vibrations, but they exhibit distinct behavior during variable speed conditions. Gear meshing vibrations are manifested by meshing frequency harmonics and associated sidebands with spacing equal to gear characteristic frequencies. These frequencies are proportional to the gearbox running speed. During variable speed processes, they follow the time-varying speed profile and change over time. For resonance vibrations, during gearbox running, sudden changes in meshing stiffness, due to gear fault and/or alternation in number of engaging gear pairs, generate impulses in load torque and further excite torsional resonance vibrations. Resonance frequencies are independent on running conditions, thus being time invariant during variable speed processes. Therefore, resonance frequencies show up as constant ones on time-frequency plane, which are parallel to the time axis but vertical to the frequency axis. On the contrary, gear meshing frequency harmonics and associated sidebands emerge as curves on time-frequency plane. According to this property, resonance frequencies can be discriminated from gear meshing frequency harmonics and associated sidebands and can be recognized as constant frequencies on time-frequency plane. Furthermore, gear fault feature can be extracted in the resonance region via sideband, amplitude, and frequency demodulation analyses.

3.2. Sensitive Component Separation. For amplitude demodulation analysis, it is necessary to separate the signal component of interest before envelope spectrum analysis. Because gear fault induced torsional vibration rides on the resonance frequency, we separate the resonance vibration component of interest using a band pass filter centered around the resonance frequency, to avoid interferences from other components.

For frequency demodulation analysis, instantaneous frequency estimation requires the signal to be monocomponent. To satisfy this requirement, we further decompose the filtered resonance component into monocomponent intrinsic mode functions (IMFs) via ensemble empirical mode decomposition (EEMD) by exploiting its capability to decompose multicomponent signal into constituent monocomponents [18]. Among the obtained IMFs, we choose the earliest IMF with an instantaneous frequency fluctuating around the resonance frequency for further frequency demodulation analysis, because (1) EEMD extracts IMFs in an order from higher to lower frequency [18, 19], (2) impulsive gear fault vibrations have significant features in higher frequency band, and (3) the signal carrier frequency is the resonance frequency.

\section{Numerical Simulation}

In this section, we illustrate the above theoretical derivations via a numerical simulated signal analysis. Considering the gear fault impulsive vibration nature in resonance region, we generate a numerical simulated signal as a series of damped sinusoids

$$
\begin{aligned}
& x(t)=\sum_{m=1}^{M} A_{m} \exp \left[-2 \pi \zeta f_{n}(t-m T)\right] \\
& \cdot \sin \left[2 \pi f_{n}(t-m T)\right] u(t-m T)+n(t),
\end{aligned}
$$

where the amplitude $A_{m}=1.5$, the damping ratio $\zeta=0.15$, the natural (resonance) frequency $f_{n}=6550 \mathrm{~Hz}$, the period 


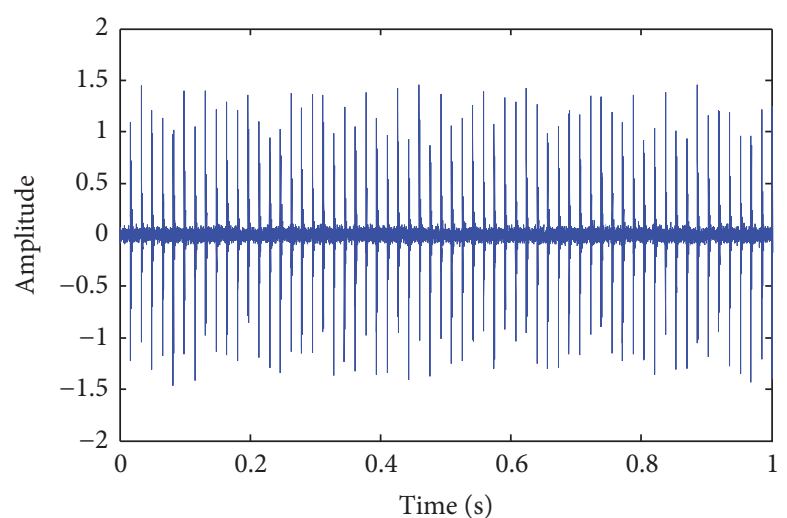

(a)

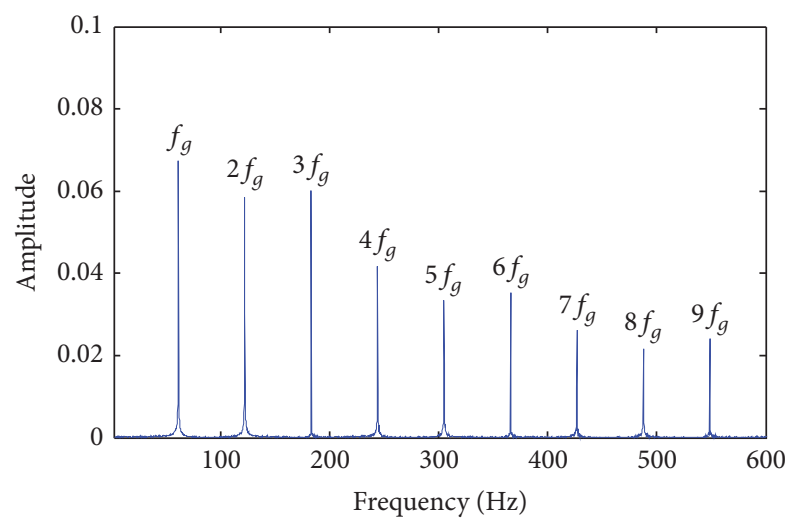

(c)

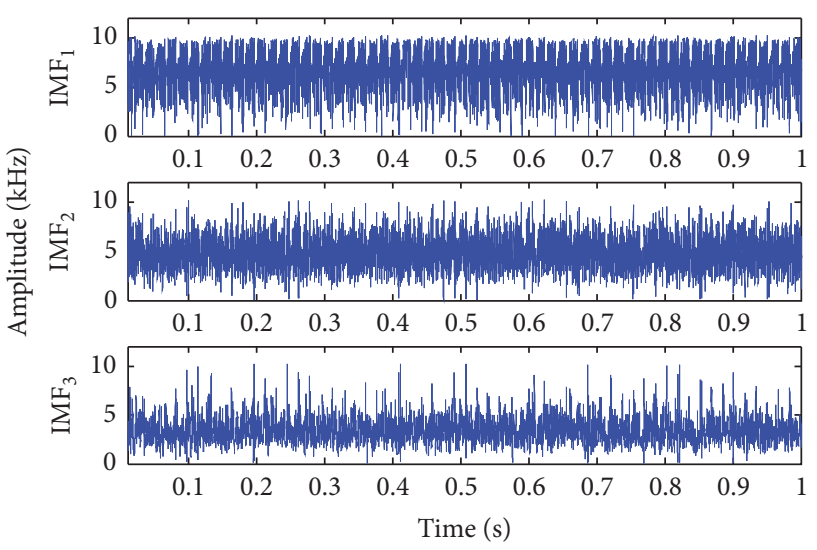

(e)

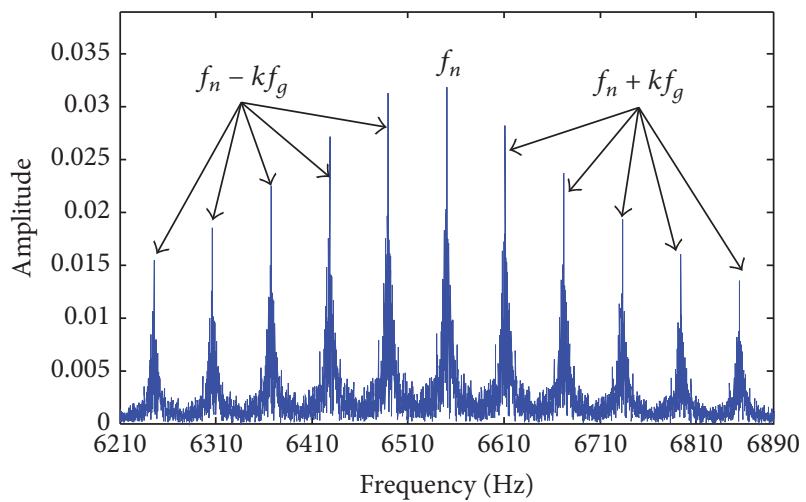

(b)
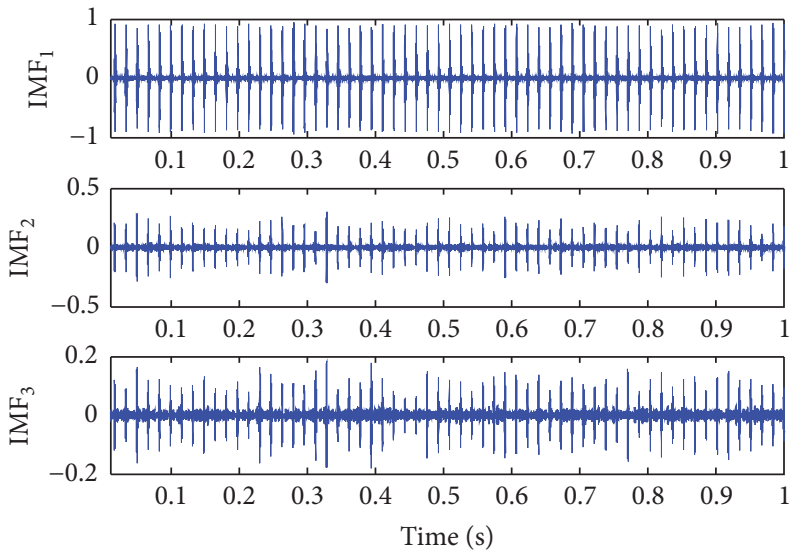

(d)

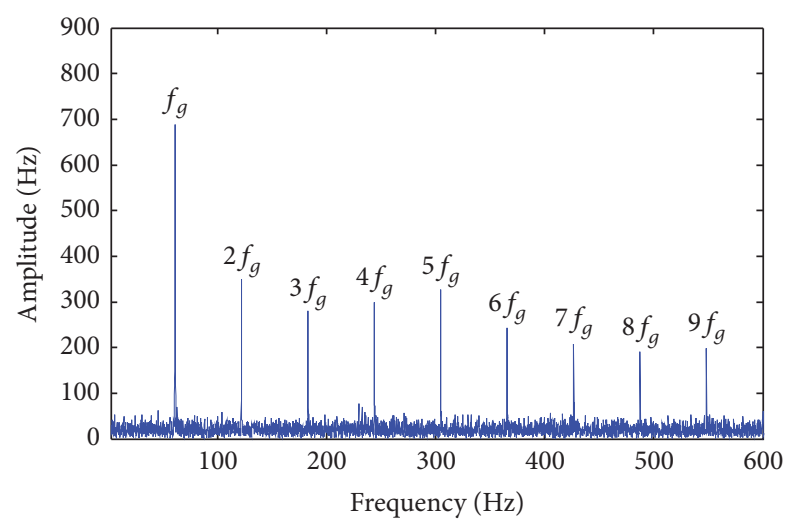

(f)

FIGURE 2: Simulated signal analysis: (a) signal waveform, (b) Fourier spectrum, (c) envelop spectrum, (d) IMFs, (e) instantaneous frequencies of IMFs, and (f) Fourier spectrum of instantaneous frequency.

of repeating impulses $T=0.0164 \mathrm{~s}$, accordingly the gear fault frequency $f_{g}=1 / T=61 \mathrm{~Hz}, u(t)$ is a unit step function, and $n(t)$ is a Gaussian white noise at a signal to noise ratio of $10 \mathrm{~dB}$ to simulate background noise.

Figures 2(a) and 2(b) show the simulated signal waveform and its Fourier spectrum, respectively. In the Fourier spectrum, peaks appear around the resonance frequency $f_{n}$, and their frequency locations correspond to the resonance frequency plus or minus the gear fault frequency harmonics $f_{n} \pm k f_{g}, k=1,2, \ldots$. These peaks form sidebands with a regular spacing equal to the gear fault frequency $f_{g}$. This behavior is consistent with the theoretical expectation from equation (4).

To avoid intricate sideband analysis, amplitude demodulation analysis is conducted. Since the simulated signal is mainly composed of resonance, bandpass filtration is omitted here. Figure 2(c) displays the envelope spectrum. The gear fault frequency harmonics $k f_{g}$ (where $k=1,2, \ldots$ ) are 


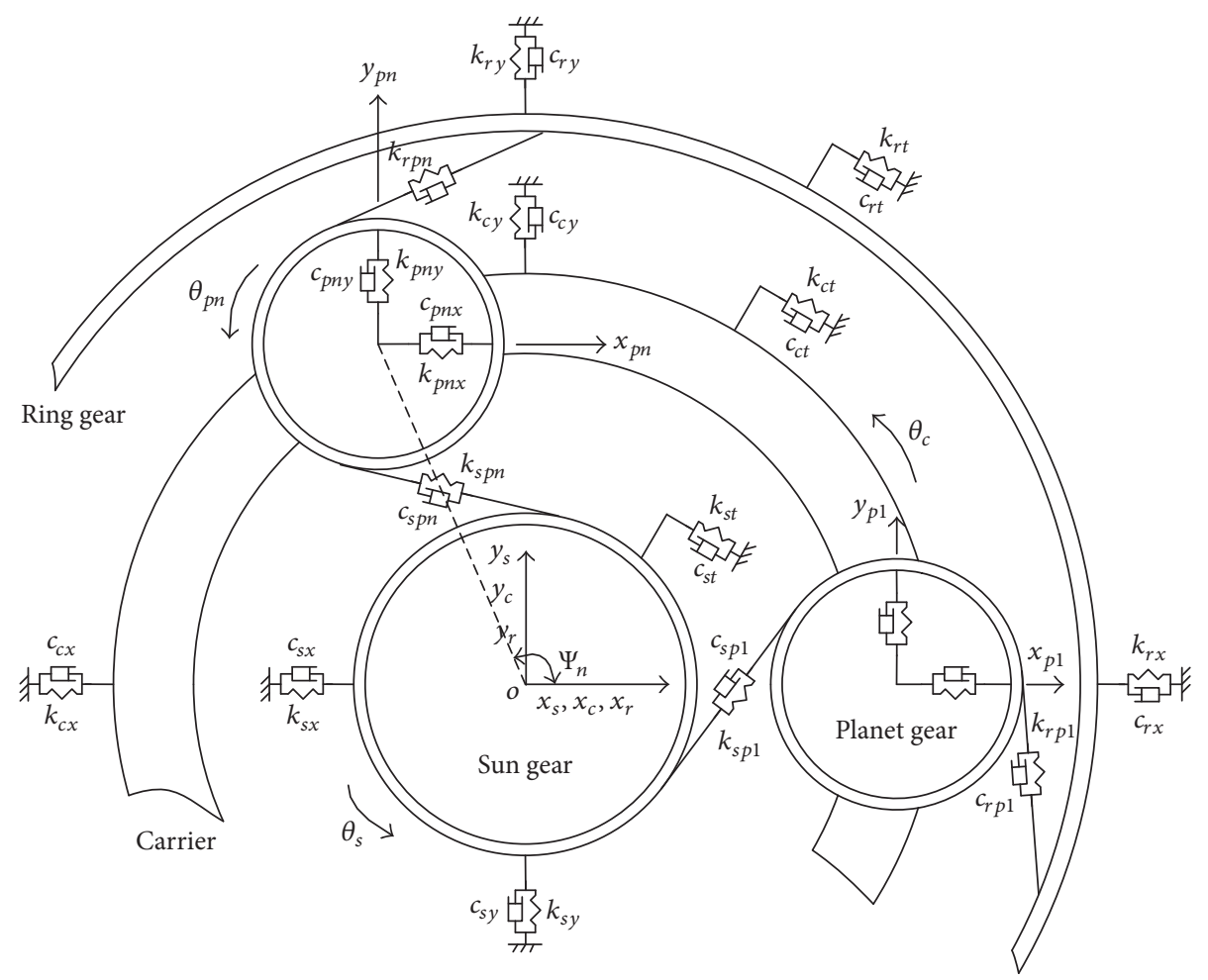

FIgURE 3: Dynamic model of a planetary gear set [16].

TABLE 1: Planetary gear set configuration.

\begin{tabular}{|c|c|c|c|c|}
\hline Parameter & Sun & Planet & Ring & Planet carrier \\
\hline Number of teeth & 19 & $31(4)$ & 81 & - \\
\hline Module (mm) & 3.2 & 3.2 & 3.2 & - \\
\hline Pressure angle $\left({ }^{\circ}\right)$ & 20 & 20 & 20 & - \\
\hline Face width $(\mathrm{m})$ & 0.0381 & 0.0381 & 0.0381 & - \\
\hline Young's modulus $(\mathrm{Pa})$ & $2.068 \times 10^{11}$ & $2.068 \times 10^{11}$ & $2.068 \times 10^{11}$ & $2.068 \times 10^{11}$ \\
\hline Poisson's ratio & 0.3 & 0.3 & 0.3 & 0.3 \\
\hline Mass (kg) & 0.7000 & 1.8220 & 5.9820 & 4.5000 \\
\hline$J / r^{2}(\mathrm{~kg})$ & 0.5292 & 1.2732 & 7.5923 & 3.0000 \\
\hline Base circle radius (m) & 0.0283 & 0.0462 & 0.1208 & 0.0745 \\
\hline Stiffness $\left(\mathrm{N} / \mathrm{m}^{2}\right)$ & \multicolumn{4}{|c|}{$k_{c}=k_{s}=k_{p}=k_{r}=1 \times 10^{9}, k_{t c}=k_{t s}=k_{t p}=0, k_{t r}=1 \times 10^{10}$} \\
\hline
\end{tabular}

Note. Number of planets in parenthesis.

dominant. They directly link to the gear fault signature, in accordance with the theoretical derivation from (6).

To avoid intricate sideband analysis in Fourier spectrum, frequency demodulation analysis is also carried out. The simulated signal is decomposed into a number of IMFs through EEMD. In EEMD, the added noise amplitude is set to 0.02 , and the ensemble number is set to 100 according to the suggestion in [19]. Figures 2(d) and 2(e) show the first three IMFs and their instantaneous frequencies, respectively. According to the proposed sensitive IMF selection criterion in Section 3.2, IMF1 is treated as the sensitive component for further analysis, since its instantaneous frequency fluctuates around the resonance frequency $6550 \mathrm{~Hz}$. Figure 2(e) presents the Fourier spectrum of sensitive IMF1 instantaneous frequency. Prominent peaks appear at the gear fault frequency harmonics $k f_{g}, k=1,2, \ldots$, directly relating to the gear fault. This feature complies with the theoretical derivation from (9).

\section{Dynamics Simulation}

In this section, we validate the theoretical derivations of gear fault signature in torsional resonance region using dynamics modelling and simulation data of a planetary gear set [16].

5.1. Gear Configuration and Dynamics Simulation. Table 1 lists a single stage planetary gear set configuration, where $J$ is 
TABLE 2: Characteristic frequencies $(\mathrm{Hz})$.

\begin{tabular}{lcccrrr}
\hline \multirow{2}{*}{ Meshing frequency } & \multicolumn{2}{c}{ Rotating frequency } & \multicolumn{3}{c}{ Fault frequency } \\
& Sun & Planet carrier & Planet & Sun & Ring \\
\hline 11.97 & 0.7778 & 0.1478 & 0.3861 & 0.7722 & 2.52 \\
\hline
\end{tabular}

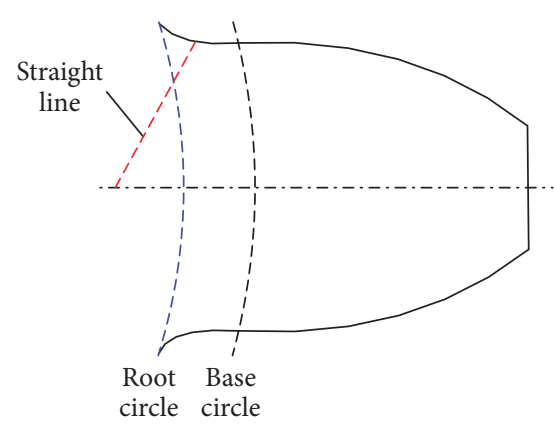

FIGURE 4: Simulated crack on sun gear tooth.

the polar moment of inertia, $K$ is the linear stiffness in lateral directions (perpendicular to the shaft axis), $K_{t}$ is the torsional stiffness, and subscripts $c, r, p$, and $s$ denote planet carrier, ring, planet, and sun gear, respectively.

Figure 3 shows the dynamic model of a planetary gear at the initial time (time zero). Using the same method of dynamics modelling and simulation, in accordance with the theoretical derivation from (11), as in [16], the first torsional resonance frequency of this planetary gear set is calculated as $f_{n}=3542.7 \mathrm{~Hz}$. To simulate gear crack fault, a crack of length $3.9 \mathrm{~mm}$ along the straight dotted line is introduced to one of the sun gear teeth, as shown in Figure 4 . The sun gear rotating frequency is set to $0.7778 \mathrm{~Hz}$. Torsional vibrations are generated and sampled at $10000 \mathrm{~Hz}$. Given the gear configuration and running speed, the planetary gear set characteristic frequencies can be calculated $[20,21]$, as listed in Table 2.

Equations of motion for the sun gear are

$$
\begin{gathered}
m_{s} \ddot{x}_{s}+c_{s x} \dot{x}_{s}+k_{s x} x_{s}+\sum F_{s p n} \cos \psi_{s n} \\
=m_{s} x_{s} \Omega^{2}+2 m_{s} \dot{y}_{s} \Omega+m_{s} y_{s} \dot{\Omega}, \\
m_{s} \ddot{y}_{s}+c_{s y} \dot{y}_{s}+k_{s y} y_{s}+\sum F_{s p n} \sin \psi_{s n} \\
=m_{s} y_{s} \Omega^{2}-2 m_{s} \dot{x}_{s} \Omega-m_{s} x_{s} \dot{\Omega}, \\
m_{s} \ddot{y}_{s}+c_{s y} \dot{y}_{s}+k_{s y} y_{s}+\sum F_{s p n} \sin \psi_{s n} \\
=m_{s} y_{s} \Omega^{2}-2 m_{s} \dot{x}_{s} \Omega-m_{s} x_{s} \dot{\Omega}, \\
\left(\frac{J_{s}}{r_{s}}\right) \ddot{\theta}_{s}+\sum F_{s p n}=\frac{T_{i}}{r_{s}},
\end{gathered}
$$

where $m, c, k$ denote mass of sun gear, damping coefficient, and stiffness of sun gear bearing; $x$ and $y$ denote the $x$ direction and $y$-direction displacement of the sun gear; $\psi_{n}$ is circumferential angle of $n$th planet; $\Omega$ is rotation speed of the carrier; $J_{s}$ and $r_{s}$ are mass moment of inertia and base circle radius of the sun gear; $T_{i}$ denotes input torque on the sun gear; $F_{s p n}$ represents the dynamic force of the $n$th sun-planet gear mesh:

$$
\begin{aligned}
F_{s p n}= & k_{s p n} \delta_{s p n}+c_{s p n} \dot{\delta}_{s p n}, \\
\delta_{s p n}= & \left(x_{s}-x_{p n}\right) \cos \psi_{s n}+\left(y_{s}-y_{p n}\right) \sin \psi_{s n}+r_{s} \theta_{s} \\
& +r_{p n} \theta_{p n}-r_{c} \theta_{c} \cos \alpha, \\
\psi_{s n}= & \frac{\pi}{2}-\alpha+\psi_{n}, \\
\psi_{n}= & \frac{2(n-1) \pi}{n},
\end{aligned}
$$

where $\alpha$ is pressure angle of gear pairs, $\delta$ is relative displacement on the lines of action, and $\theta$ is angular displacement.

5.2. Signal Analysis. Figure 5 shows the signal analysis result. In the Fourier spectrum, Figure 5(b), we concentrate on the resonance region around $f_{n}=3542.7 \mathrm{~Hz}$ to extract gear fault signature through sideband analysis. In addition to those at $f_{n}-3 f_{s}+4 f_{c}$ and $f_{n}-5 f_{s}+4 f_{c}$, most peaks appear at $f_{n} \pm k f_{s}$, and they form sidebands with a regular spacing equal to the sun gear fault frequency $f_{s}$. To separate the resonance component for further demodulation analysis, the raw signal is band pass filtered around the resonance frequency $f_{n}=3542.7 \mathrm{~Hz}$ with a bandwidth of $300 \mathrm{~Hz}$. Figure 5(c) displays the envelope spectrum. Most of dominant peaks correspond to the sun gear fault frequency harmonics $k f_{s}$, besides $5 f_{s}-4 f_{c}$ and $10 f_{s}-4 f_{c}$. Several IMFs are generated through EEMD of the separated resonance component. In EEMD, the added noise amplitude is set to 0.02 , and the ensemble number is set to 100 , following the suggestion in [19]. Among them, 


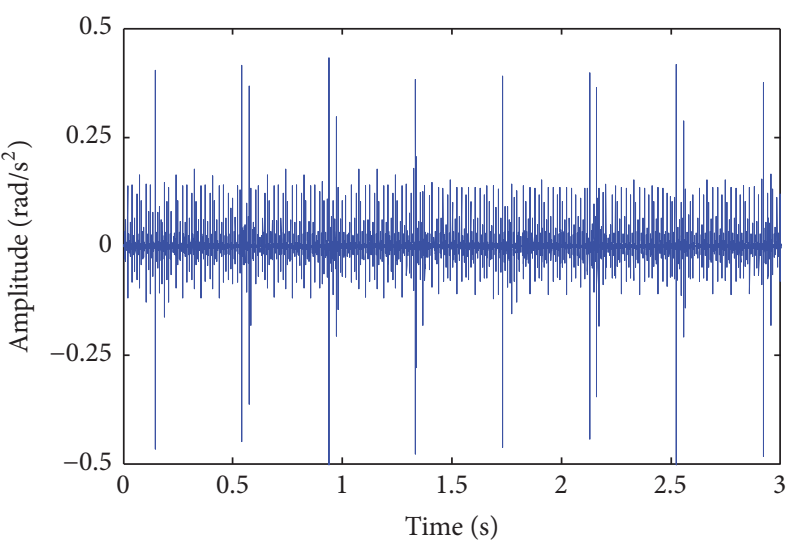

(a)

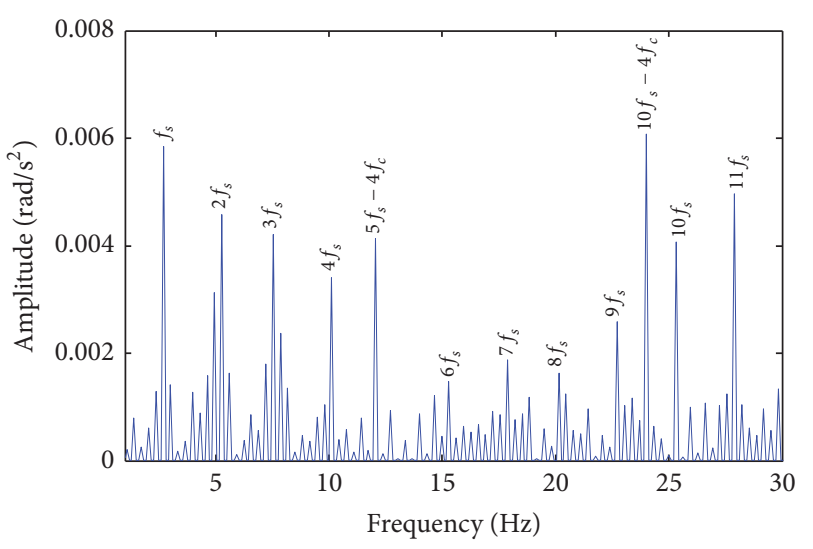

(c)

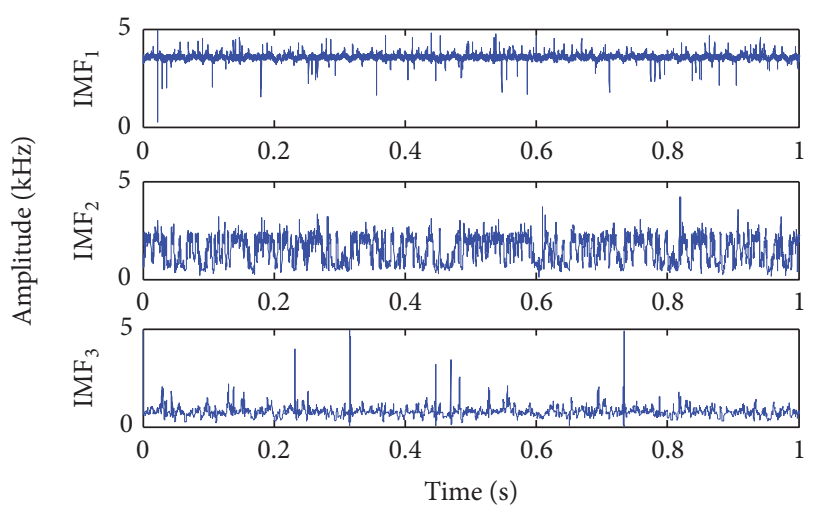

(e)

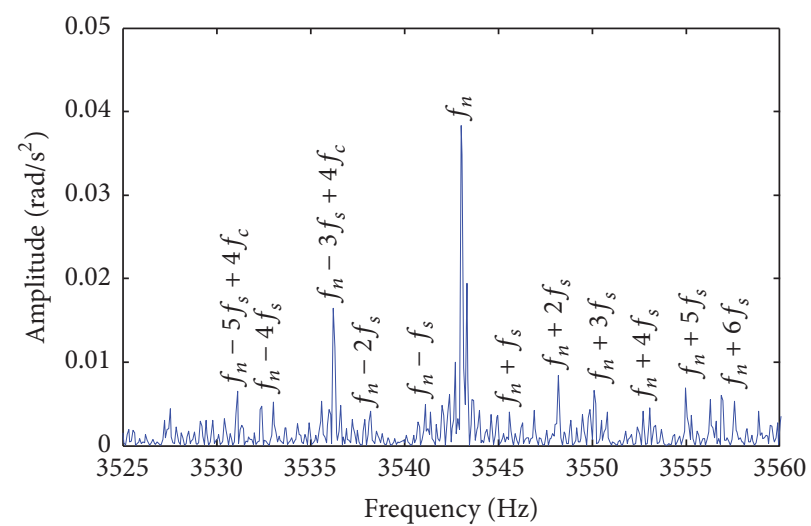

(b)

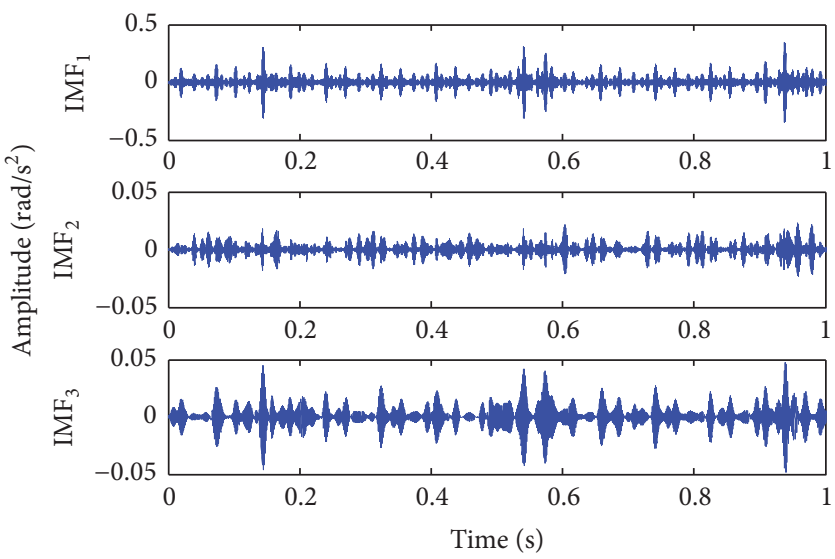

(d)

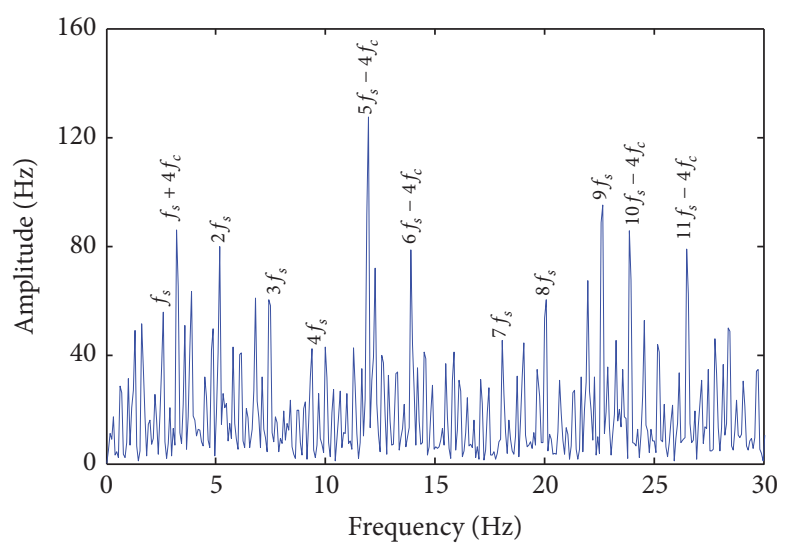

(f)

FIGURE 5: Dynamics modelling signal analysis: (a) torsional vibration signal, (b) Fourier spectrum, (c) envelop spectrum, (d) IMFs, (e) instantaneous frequencies of IMFs, and (f) Fourier spectrum of instantaneous frequency.

IMF1 has an instantaneous frequency fluctuating around the resonance frequency $f_{n}=3542.7 \mathrm{~Hz}$ and hence is selected for frequency demodulation analysis. Figure 5(f) shows the Fourier spectrum of IMF1 instantaneous frequency. Although the sun gear fault frequency harmonics plus or minus four times the planet carrier rotating frequency $k f_{s} \pm 4 f_{c}$ dominate, the sun gear fault frequency harmonics $k f_{s}$ also have prominent magnitudes. In the above Fourier spectrum, and amplitude and frequency demodulated spectra, all the peaks relate to the sun gear fault frequency $f_{s}$. These findings imply the sun gear fault, in accordance with actual settings in dynamics modelling. 


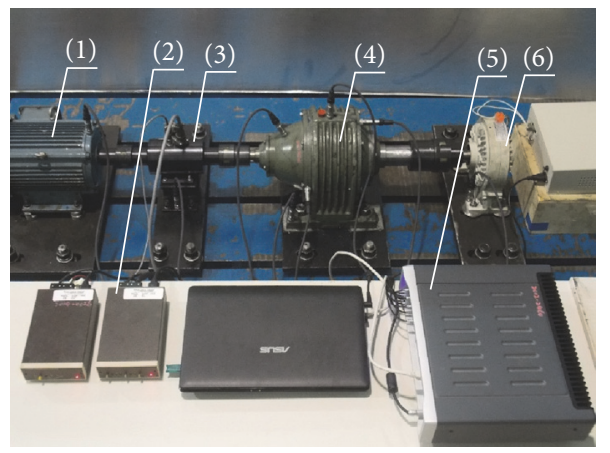

FIGURE 6: Planetary gearbox test rig: (1) drive motor, (2) signal conditioner, (3) torque-speed transducer, (4) planetary gearbox, (5) signal collector, and (6) magnetic powder brake.
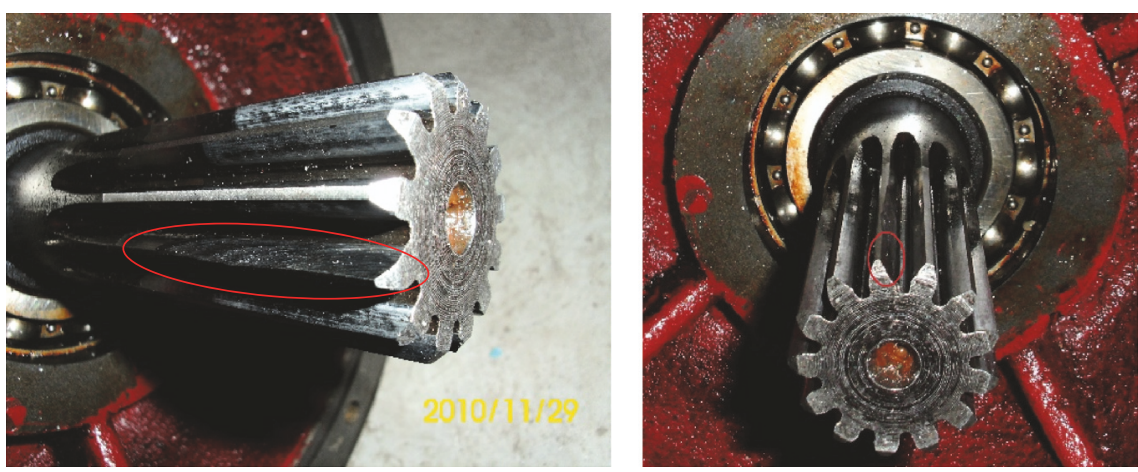

(a)
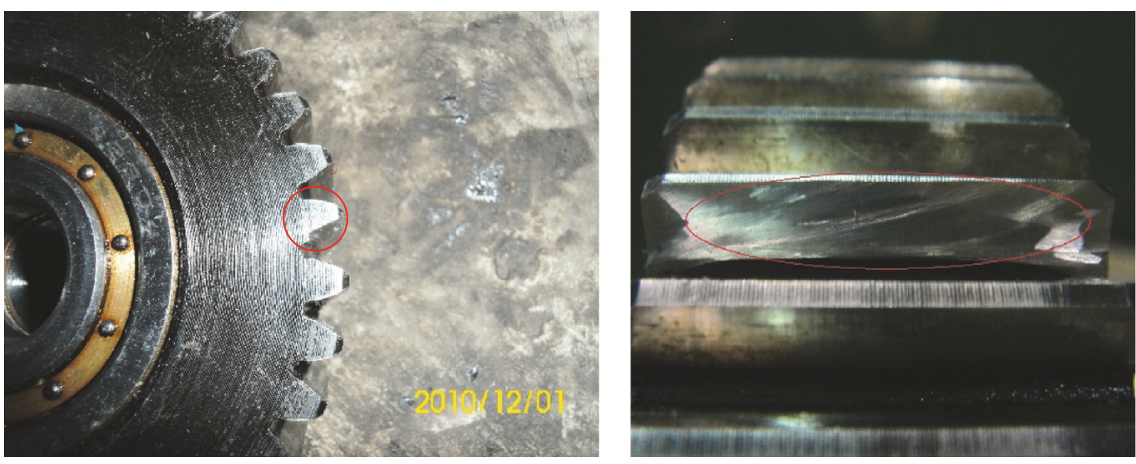

(b)
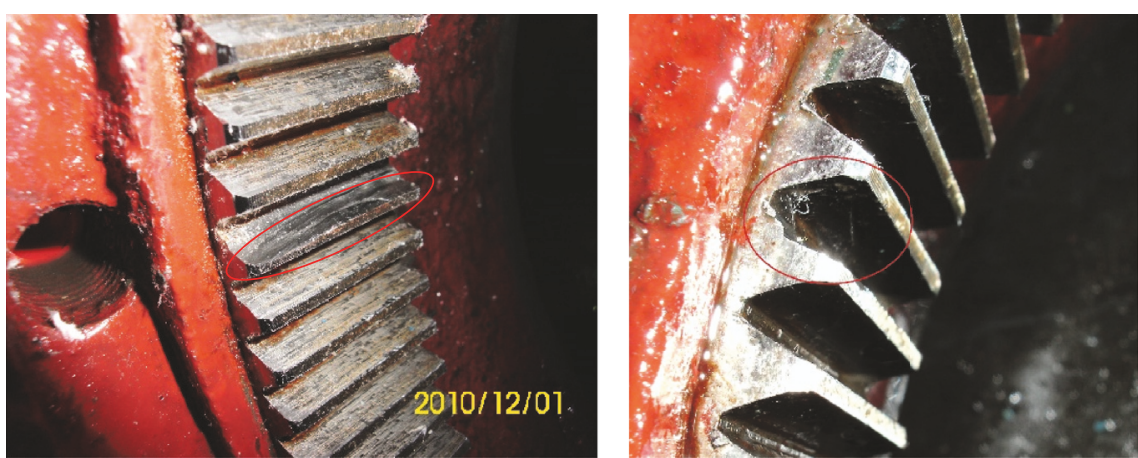

(c)

Figure 7: Localized fault on (a) sun, (b) planet, and (c) ring gear. 


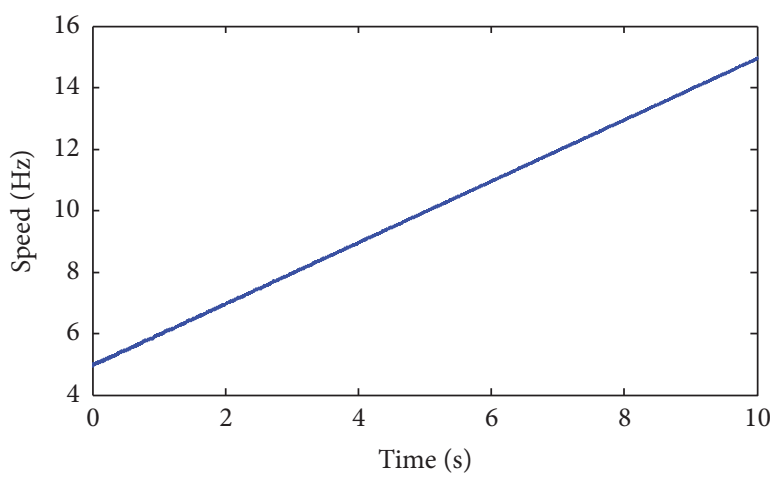

(a)

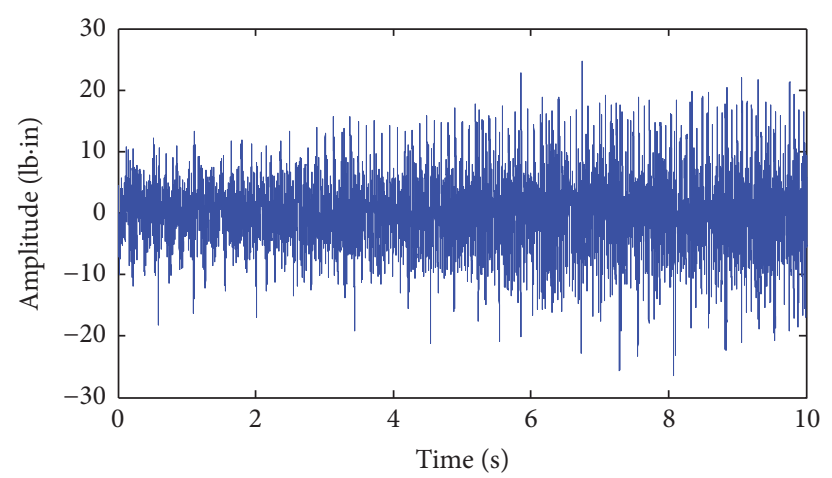

(b)

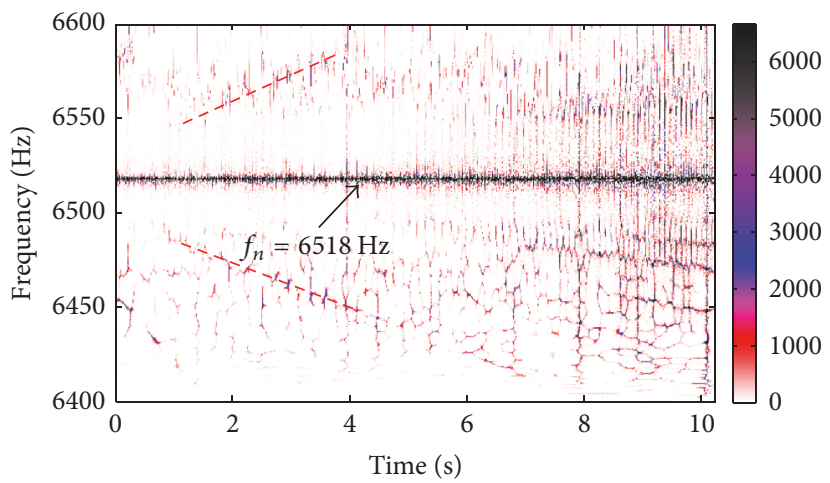

(c)

FIGURE 8: Resonance frequency identification: (a) drive motor speed, (b) detrended torsional vibration signal, and (c) reassigned Morlet scalogram.

TABLE 3: Gear configuration parameters.

\begin{tabular}{lccc}
\hline Gear & Sun & Planet & Ring \\
\hline Number of gear teeth & 13 & $38(3)$ & 92 \\
\hline
\end{tabular}

Note. Number of planet gears in parentheses.

\section{Experimental Validation}

In this section, we validate the proposed idea using lab experimental signals of a planetary gearbox under healthy and faulty gear conditions, respectively.

6.1. Experimental Setting. Figure 6 shows the planetary gearbox test rig. Table 3 lists the gearbox configuration parameters. The planetary gearbox is driven by a drive motor through the sun gear shaft, and a load of $25 \mathrm{lb} \cdot$ in is applied by a magnetic powder brake via the planet carrier shaft. A speed-torque transducer is installed between the drive motor and the planetary gearbox. The torque sensor we used is an integrated sensor, and its output is torsional vibration signal only. During experiments, the torque (torsional vibration) and speed are collected at $20480 \mathrm{~Hz}$.

To simulate localized gear fault, one tooth of the sun, one planet, and ring gear are locally chipped, respectively, as shown in Figure 7. Four types of tests are conducted: baseline case when all gears are healthy and faulty sun, planet, and ring gear case when the sun, one planet, and the ring gear alone are locally chipped, respectively. For each case, two running speed conditions are set. One is a constant speed condition to be used for fault feature extraction, when the drive motor speed is set to $23.5 \mathrm{~Hz}$. The other is a variable speed condition for resonance frequency identification, when the drive motor speeds up linearly.

Given the gearbox configuration parameters and running speed, gear characteristic frequency can be calculated $[20,21]$, as listed in Table 4.

6.2. Signal Analysis. In this section, we firstly identify the resonance frequency via time-frequency analysis of torsional vibration signals during speed-up processes. Then, we extract gear fault signature through sideband analysis in Fourier spectrum within resonance region, and amplitude and frequency demodulation analysis of sensitive resonance component. For demodulation analysis, we separate the component of interest using a bandpass filter with a center frequency equal to the identified resonance frequency and a bandwidth of $200 \mathrm{~Hz}$. In EEMD, the added noise amplitude is set to 0.02 , and the ensemble number is 100 according to the suggestion in [19]. 


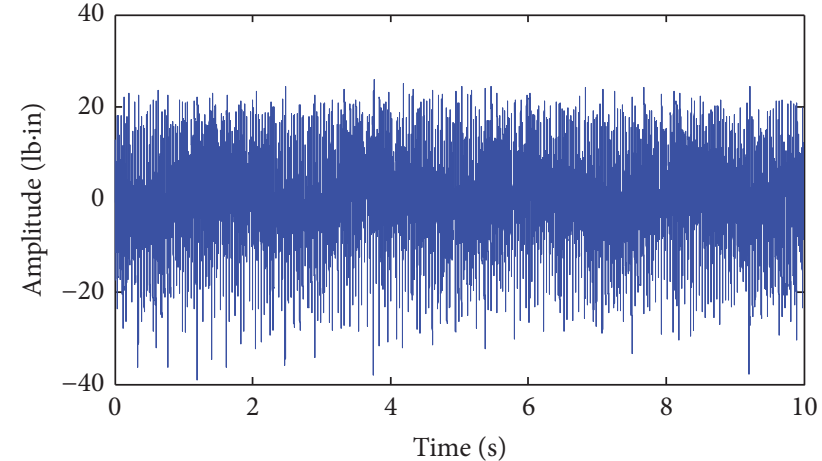

(a)

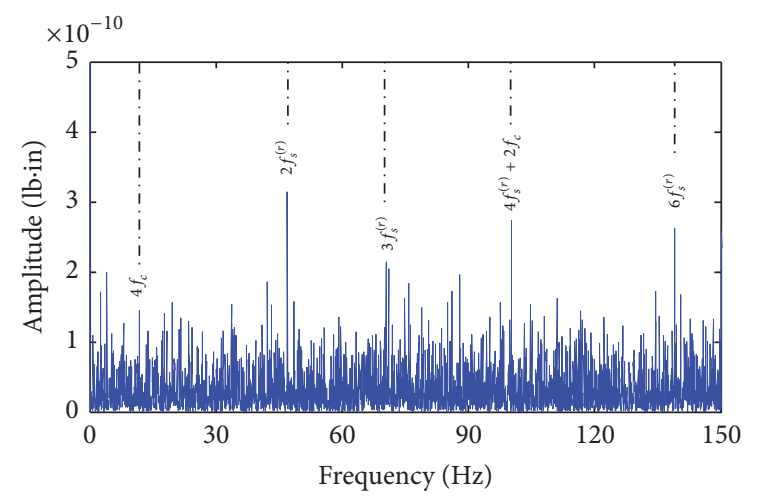

(c)

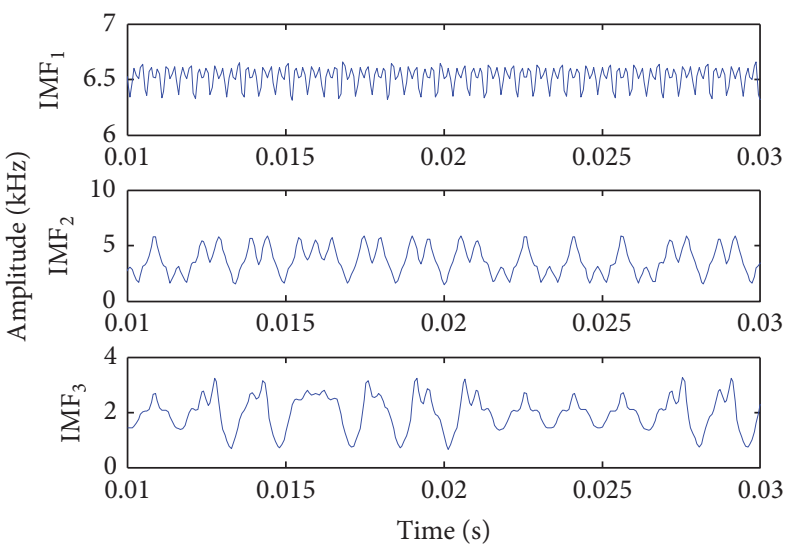

(e)

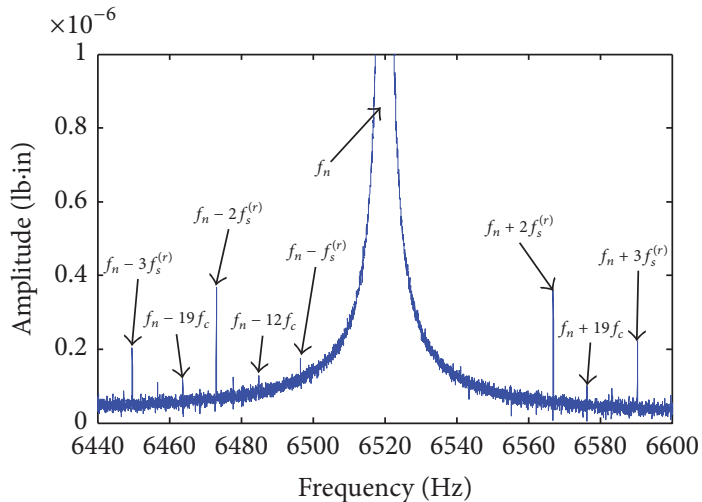

(b)

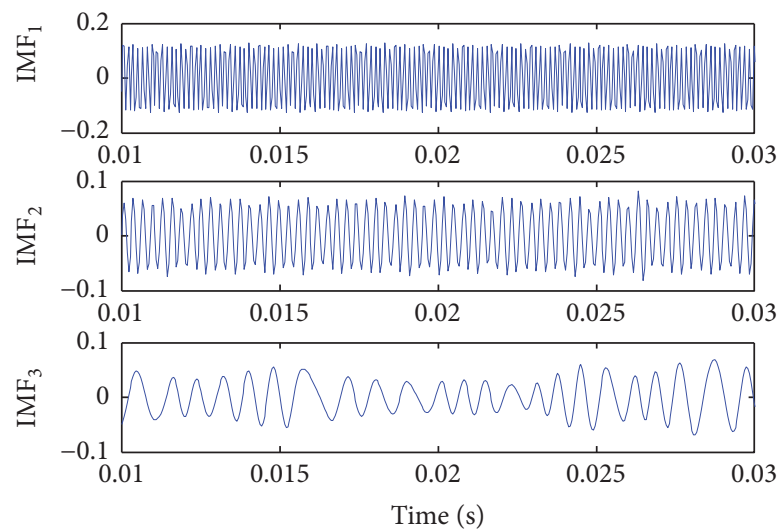

(d)

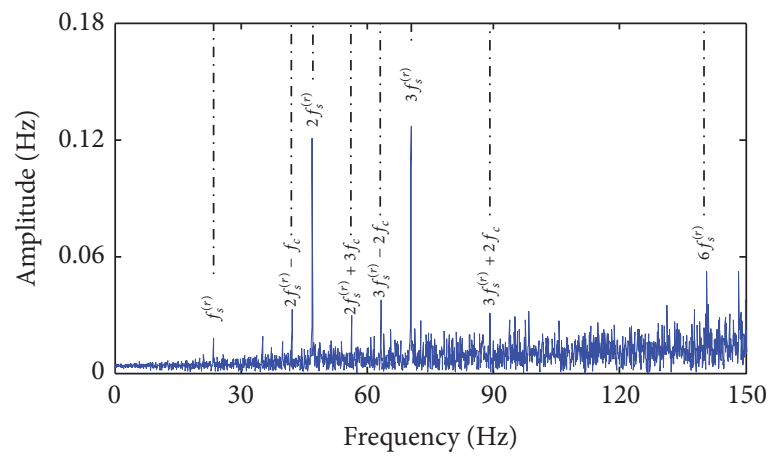

(f)

Figure 9: Baseline signal analysis: (a) detrended torsional vibration signal, (b) Fourier spectrum, (c) envelop spectrum, (d) IMFs, (e) instantaneous frequencies of IMFs, and (f) Fourier spectrum of instantaneous frequency.

TABLE 4: Gearbox characteristic frequencies $(\mathrm{Hz})$.

\begin{tabular}{lccccc}
\hline \multirow{2}{*}{ Meshing frequency } & \multicolumn{2}{c}{ Rotating frequency } & \multicolumn{2}{c}{ Fault frequency } \\
& Sun gear & Planet carrier & Sun gear & Planet gear & Ring gear \\
\hline 267.676 & 23.5 & 2.91 & 61.77 & 7.044 & 8.73 \\
\hline
\end{tabular}




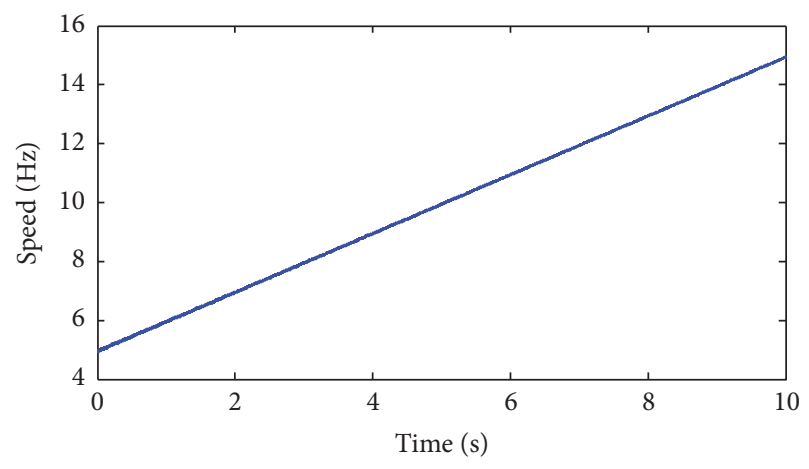

(a)

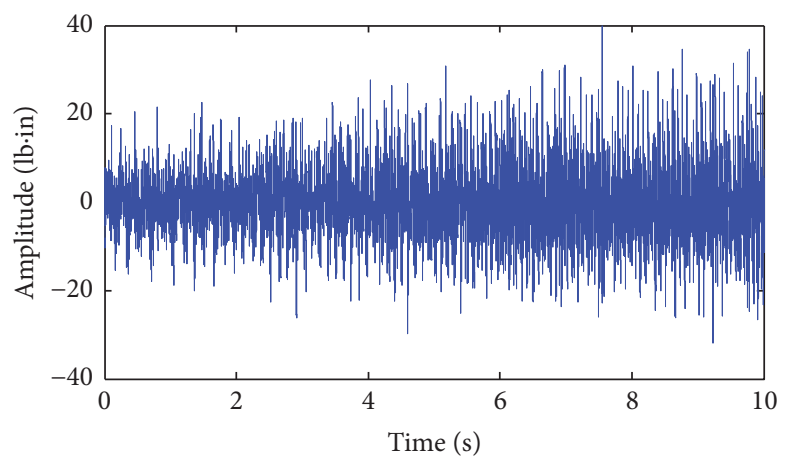

(b)

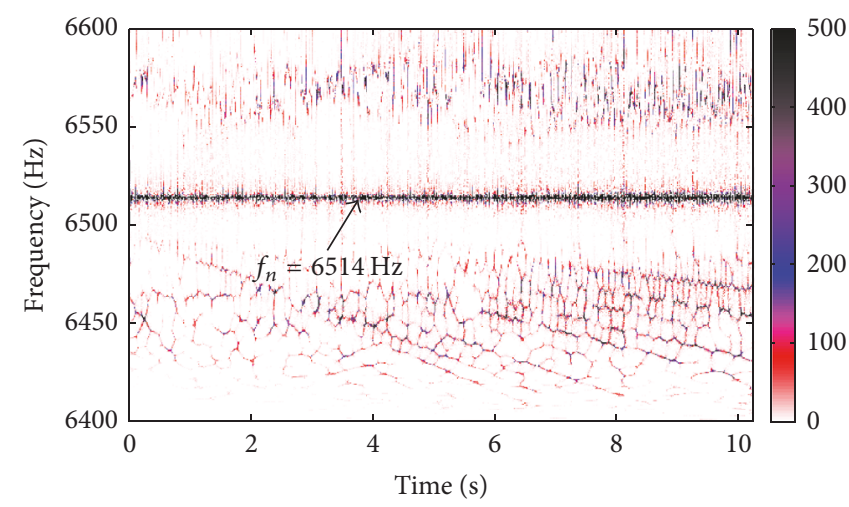

(c)

FIGURE 10: Resonance frequency identification: (a) drive motor speed, (b) detrended torsional vibration signal, and (c) reassigned Morlet scalogram.

6.2.1. Baseline. In the baseline case, a speed-up test is conducted for resonance frequency identification via timefrequency analysis. The drive motor speeds up from $5 \mathrm{~Hz}$ to $15 \mathrm{~Hz}$ in 10 seconds approximately. Figures 8(a), 8(b), and $8(\mathrm{c})$ show the speed, torsional vibration signal, and its reassigned Morlet scalogram, respectively. In Figure 8(c), within the frequency band $[6400,6600] \mathrm{Hz}$, the dominant frequency is constant and corresponds to $6518 \mathrm{~Hz}$. Other frequencies change over time. The frequencies higher than $6518 \mathrm{~Hz}$ increase in direct proportionality to the variable speed, while those lower than $6518 \mathrm{~Hz}$ decrease in inverse proportionality to the variable speed. They form a dispersive sideband structure symmetric about $6518 \mathrm{~Hz}$, as indicated by red dash lines. According to this feature, $6518 \mathrm{~Hz}$ is treated as a resonance frequency for further sideband analysis in Fourier spectrum, and amplitude and frequency demodulation analysis under constant speed conditions.

Figures 9(a) and 9(b) show the torsional vibration signal and its Fourier spectrum under constant speed. In Fourier spectrum, sidebands appear around the resonance frequency, such as the resonance frequency plus or minus the planet carrier and sun gear rotating frequency harmonics $f_{n} \pm k f_{c}$, $f_{n} \pm l f_{s}^{(r)}$, where $k, l=1,2, \ldots$. This does not indicate any gear fault, because gearbox manufacturing or assembling errors will generate torque fluctuation at the sun gear and planet carrier shaft rotating frequencies, resulting in presence of the sidebands.
For amplitude demodulation analysis, we separate the component of interest using a bandpass filter. Figure 9(c) displays the envelope spectrum of separated resonance component. Prominent peaks exist at the planet carrier rotating frequency harmonics $k f_{c}$, the sun gear rotating frequency harmonics $l f_{s}^{(r)}$, and their combinations $l f_{s}^{(r)} \pm k f_{c}$, such as $4 f_{c}, 2 f_{s}^{(r)}, 3 f_{s}^{(r)}, 6 f_{s}^{(r)}$, and $4 f_{s}^{(r)}+2 f_{c}$. For frequency demodulation analysis, we decompose the separated resonance component via EEMD and calculate their instantaneous frequencies, as shown in Figures 9(d) and 9(e). According to the sensitive IMF selection criterion, we choose IMF1 for further analysis, since its instantaneous frequency fluctuates around the resonance frequency. Figure 9(f) presents the frequency demodulated spectra. Peaks emerge at the sun gear rotating frequency harmonics $l f_{s}^{(r)}$, their combinations with the planet carrier rotating frequency harmonics $l f_{s}^{(r)} \pm k f_{c}$, such as $(1,2,3,6) f_{s}^{(r)}, 2 f_{s}^{(r)}-f_{c}, 2 f_{s}^{(r)}+3 f_{c}, 3 f_{s}^{(r)}-2 f_{c}$, and $3 f_{s}^{(r)}+2 f_{c}$. In both amplitude and frequency demodulated spectra, the peaks do not link to any gear fault frequency or their harmonics. As such, they do not imply fault existence on any gear.

6.2.2. Sun Gear Fault. Figure 10 shows the time-frequency analysis result of torsional vibration signal during a speedup process under sun gear fault. In this case, the resonance frequency is identified as $6514 \mathrm{~Hz}$, according to its time 


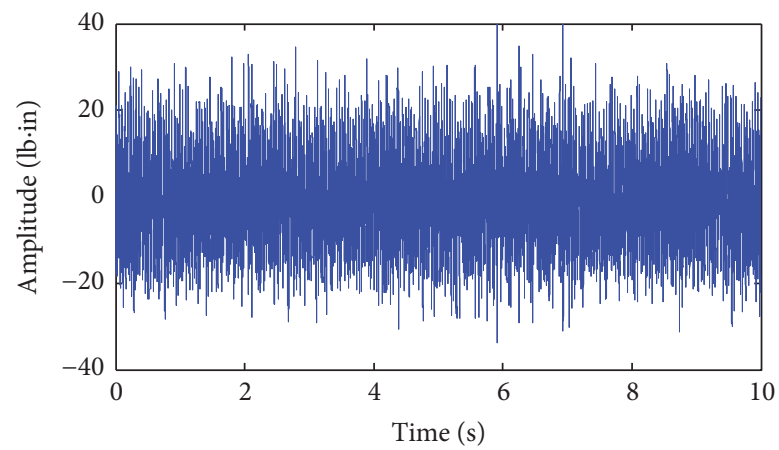

(a)

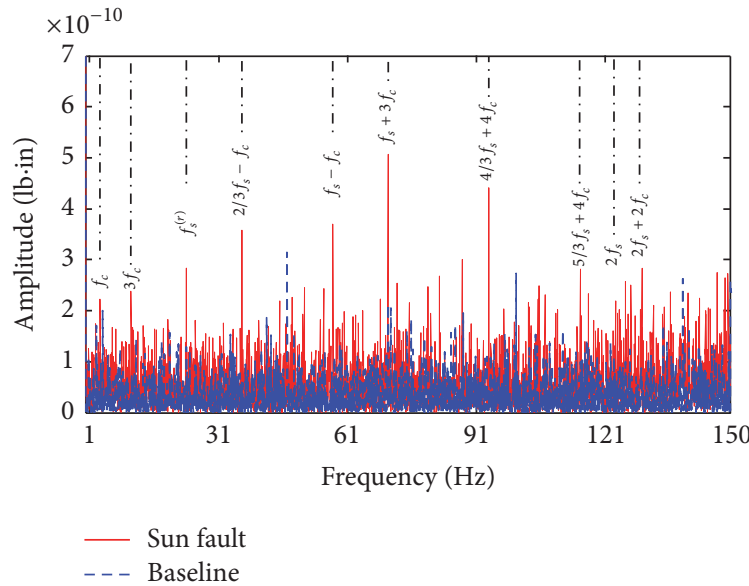

(c)

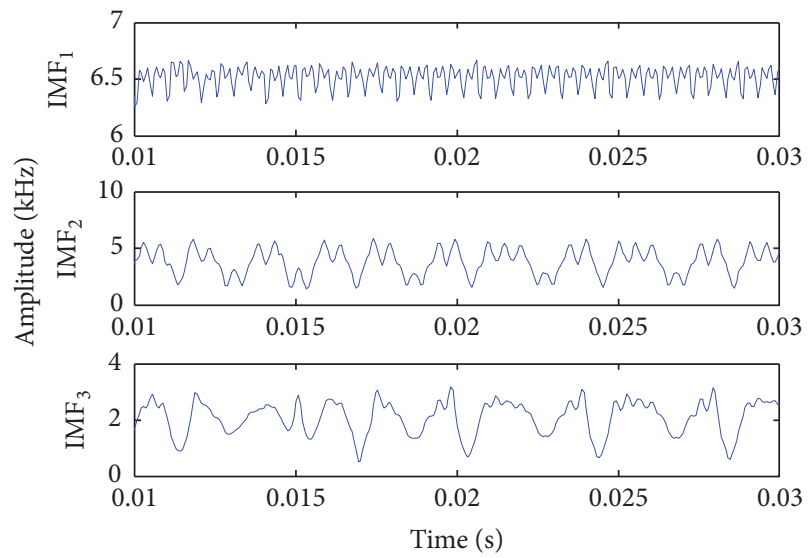

(e)

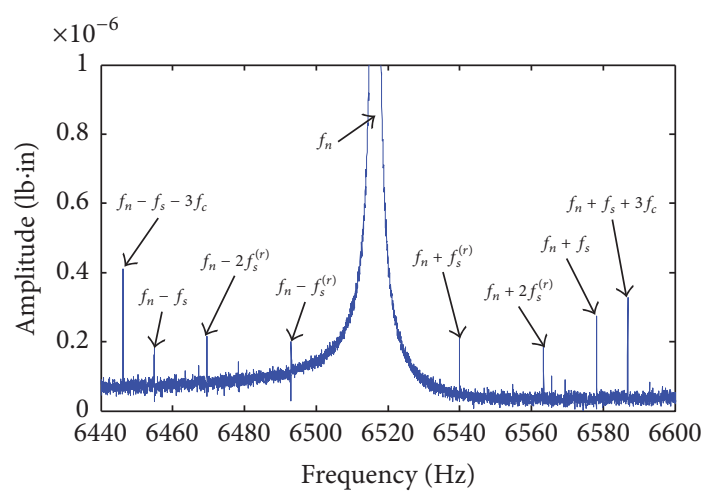

(b)
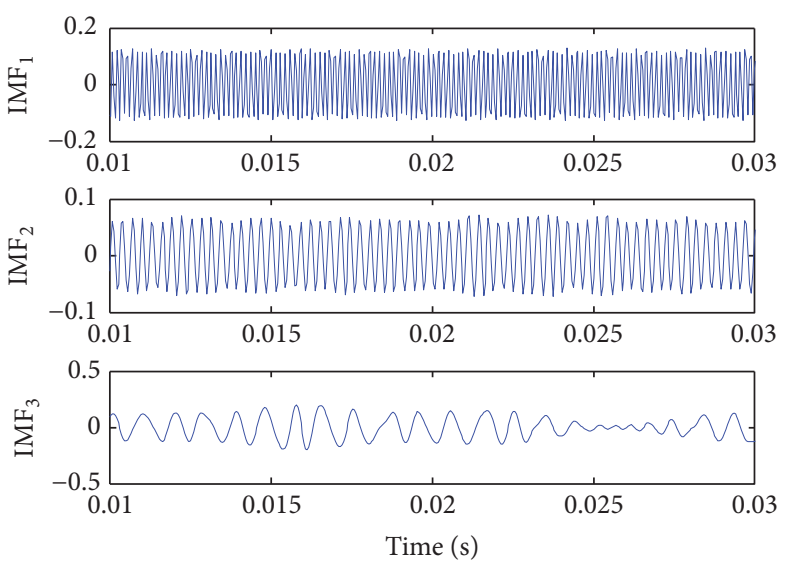

(d)

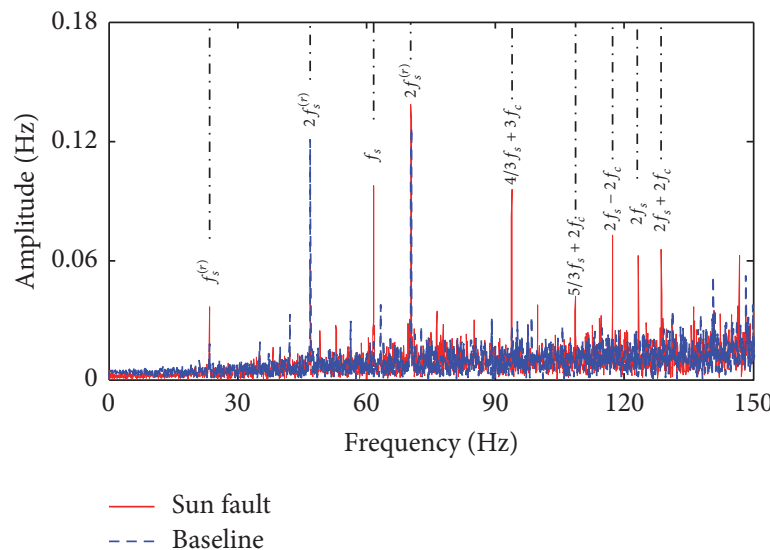

(f)

FIGURE 11: Sun gear fault signal analysis: (a) detrended torsional vibration signal, (b) Fourier spectrum, (c) envelop spectrum, (d) IMFs, (e) instantaneous frequencies of IMFs, and (f) Fourier spectrum of instantaneous frequency.

invariant nature under variable speeds; see Figure 10(c). The identified resonance frequency differs somewhat from that in the baseline case, since the gearbox dynamic property might change after replacing a gear.

Figure 11 displays the Fourier spectrum, and amplitude and frequency demodulation analysis results. In the Fourier spectrum, Figure 11(b), in addition to the sidebands corresponding to the sun gear rotating frequency $f_{n} \pm l f_{s}^{(r)}$, $l=1,2, \ldots$, new sidebands emerge. They correspond to the resonance frequency plus or minus the sun gear fault frequency $f_{n} \pm f_{s}$, and the resonance frequency plus or minus combination (sum or difference) of the sun gear 


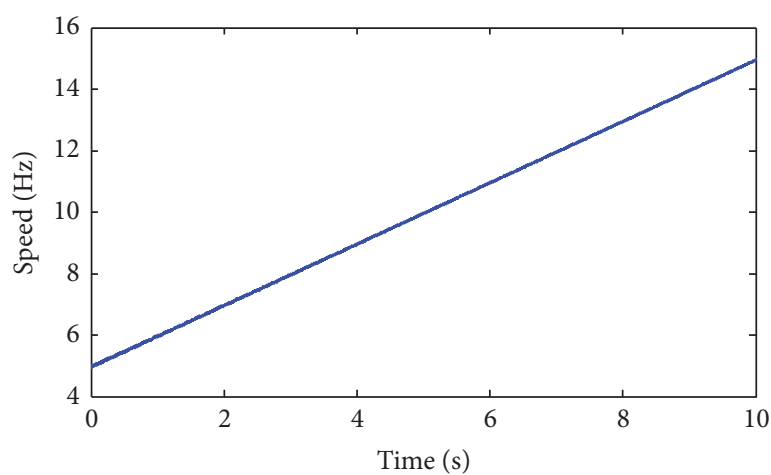

(a)

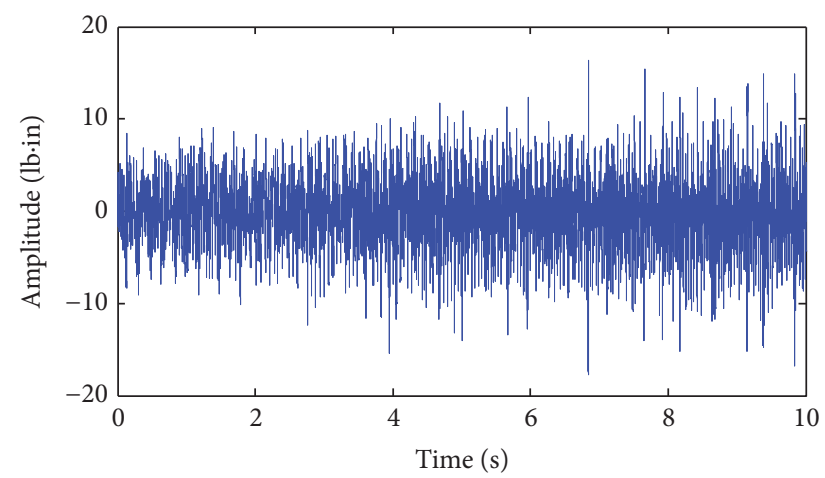

(b)

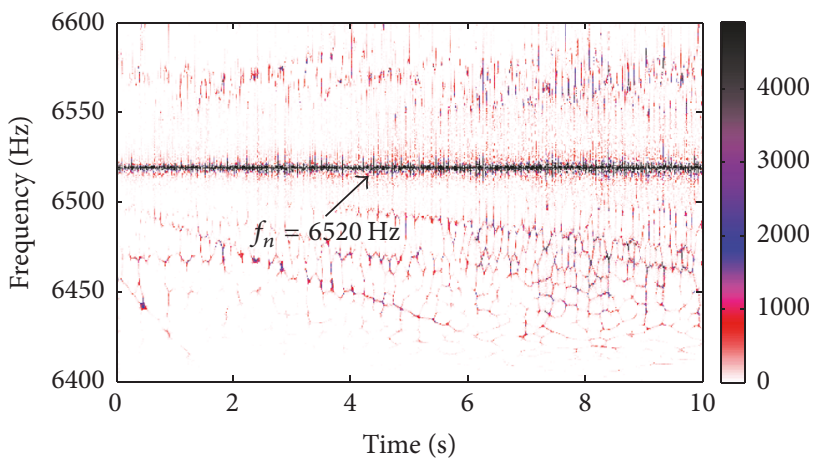

(c)

Figure 12: Resonance frequency identification: (a) drive motor speed, (b) detrended torsional vibration signal, and (c) reassigned Morlet scalogram.

fault frequency and three times the planet carrier rotating frequency $f_{n} \pm f_{s} \pm 3 f_{c}$. The expressions in these new sidebands all have a common term equal to the sun gear fault frequency, indicating existence of the sun gear fault.

In the envelope spectrum of filtered resonance component, the sun gear rotating frequency and the planet carrier rotating frequency harmonics still exist. Moreover, pronounced peaks also appear at $1 / 3$ harmonics of the sun fault frequency plus or minus the planet carrier rotating frequency harmonics $1 / 3 n f_{s} \pm k f_{c}$. In the Fourier spectrum of sensitive IMF1 instantaneous frequency, Figure 11(f), the sun gear rotating frequency and its harmonics $l f_{s}^{(r)}, l=1,2, \ldots$, also show up. Meanwhile, prominent peaks appear at the sun gear fault frequency and its harmonics (such as $f_{s}$ and $2 f_{s}$ ), as well as $1 / 3$ harmonics of the sun fault frequency plus or minus the planet carrier rotating frequency harmonics (e.g., $4 / 3 f_{s}+3 f_{c}, 5 / 3 f_{s}+2 f_{c}, 2 f_{s}-2 f_{c}$, and $2 f_{s}+2 f_{c}$ ). These peaks are higher than those of the baseline signal, and they relate to the sun gear fault frequency, indicating the sun gear fault. Due to manufacturing error, planet gears are not perfectly identical. When they mesh with the faulty sun gear tooth, the generated fault impulses differ among planet gears. In this case, the sun gear fault frequency can be modified by dividing by the number of planet gears 3 . This is why $1 / 3$ harmonics of the sun fault frequency are present.
6.2.3. Planet Gear Fault. In this case, the resonance frequency is recognized as $6520 \mathrm{~Hz}$; see the time-frequency analysis of torsional vibration signal during a speed-up process, Figure 12(c).

The sideband around the resonance frequency in Fourier spectrum is exhibited in Figure 13(b). In addition to the sidebands related to the sun gear rotating frequency $f_{n} \pm l f_{s}^{(r)}$, $l=1,2, \ldots$, prominent peaks exist at the resonance frequency plus or minus the planet gear fault frequency harmonics, such as $f_{n}-6 f_{p}, f_{n}+3 f_{p}$, and $f_{n} \pm n f_{p}, n=1,2, \ldots$.

In the envelope spectrum of filtered resonance component, Figure 13(c), the planet gear fault frequency harmonics $n f_{p}$ (e.g., $2 f_{p}$ and $4 f_{p}$ ) and their sum or difference combination with the planet carrier rotating frequency harmonics $n f_{p} \pm k f_{c}$ are present. In the Fourier spectrum of sensitive IMF1 instantaneous frequency, Figure 13(f), although the sun gear rotating frequency $f_{s}^{(r)}$ is dominant, prominent peaks emerge at the planet fault frequency harmonics $n f_{p}$, and their sum or difference combinations with the planet carrier rotating frequency harmonics $n f_{p} \pm k f_{c}$. These peaks in both amplitude and frequency demodulation spectra relate to the planet gear fault frequency, and they are higher than the baseline signal, implying fault existence on one planet gear.

6.2.4. Ring Gear Fault. In this case, the resonance frequency is discerned as $f_{n}=6512 \mathrm{~Hz}$, through the time-frequency 


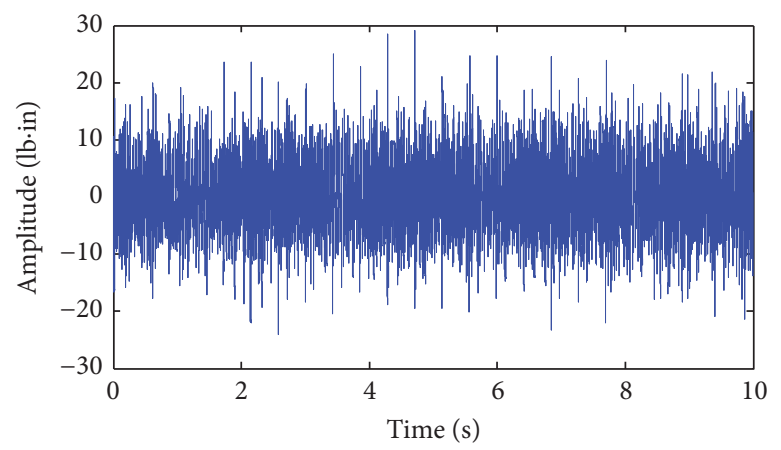

(a)

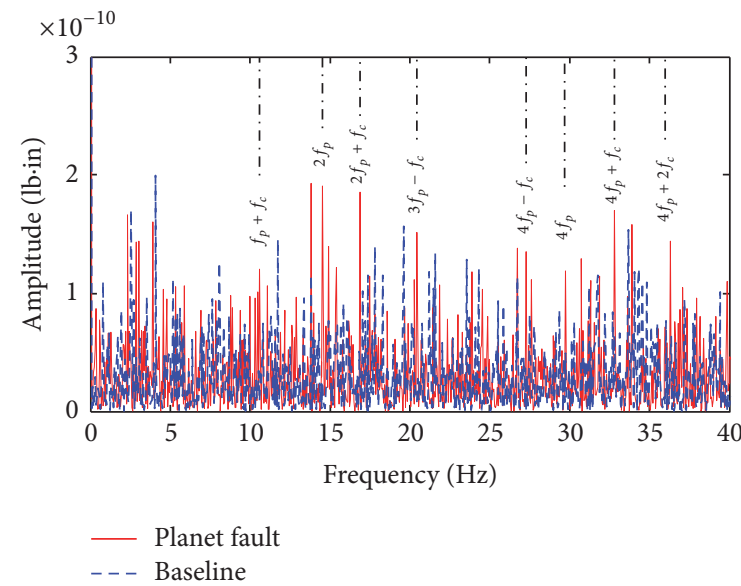

(c)

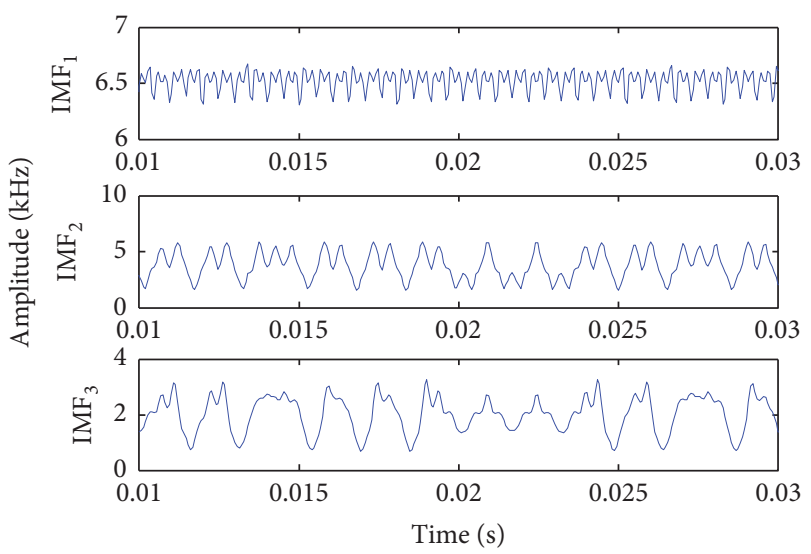

(e)

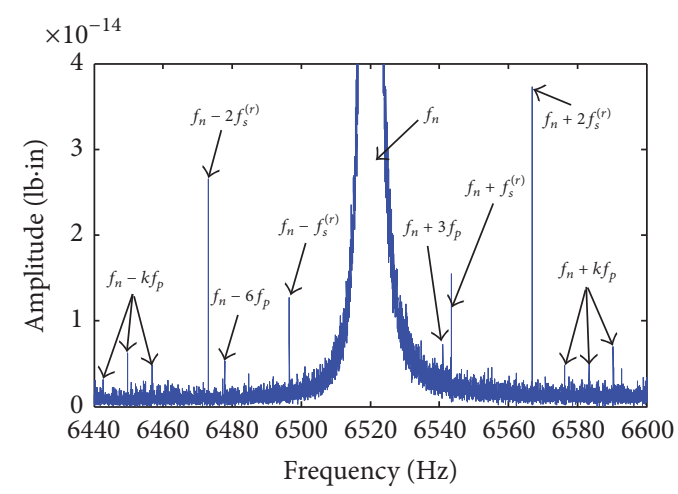

(b)
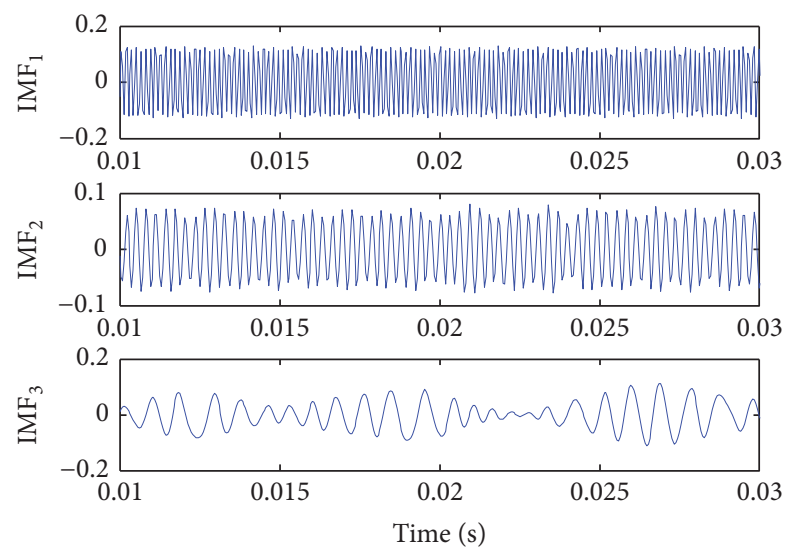

(d)

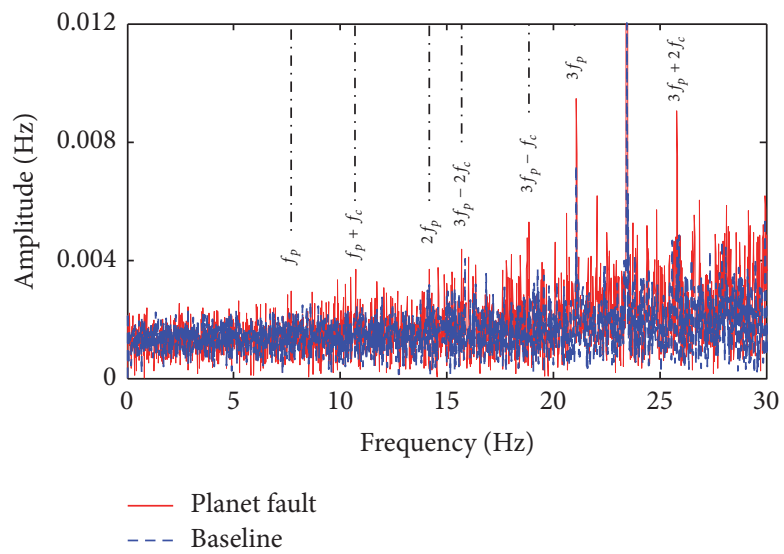

(f)

FIGURE 13: Planet gear fault signal analysis: (a) detrended torsional vibration signal, (b) Fourier spectrum, (c) envelop spectrum, (d) IMFs, (e) instantaneous frequencies of IMFs, and (f) Fourier spectrum of instantaneous frequency.

analysis of torsional vibration signal during a speed-up process; see Figure 14(c).

In the Fourier spectrum around the resonance frequency $f_{n}=6512 \mathrm{~Hz}$, Figure 15(b), sidebands relevant to the ring gear fault frequency $f_{r}$ or its $1 / 3$ harmonics $1 / 3 f_{r}$ show up (such as $f_{n} \pm 4 f_{r}, f_{n} \pm 19 / 3 f_{r}$, and $f_{n}-7 f_{r}$ ), in addition to those associated with the sun gear rotating frequency.
In the envelope spectrum of filtered resonance component, Figure 15(c), dominant peaks appear at $1 / 3$ harmonics of ring gear fault frequency $1 / 3 n f_{r}, n=1,2, \ldots$. In the Fourier spectrum of sensitive IMF1 instantaneous frequency, Figure 15(f), except the dominant peaks at three times the planet gear fault frequency $3 f_{p}$ and the sun gear rotating frequency $f_{s}^{(r)}$, all the prominent peaks correspond to $1 / 3$ harmonics 


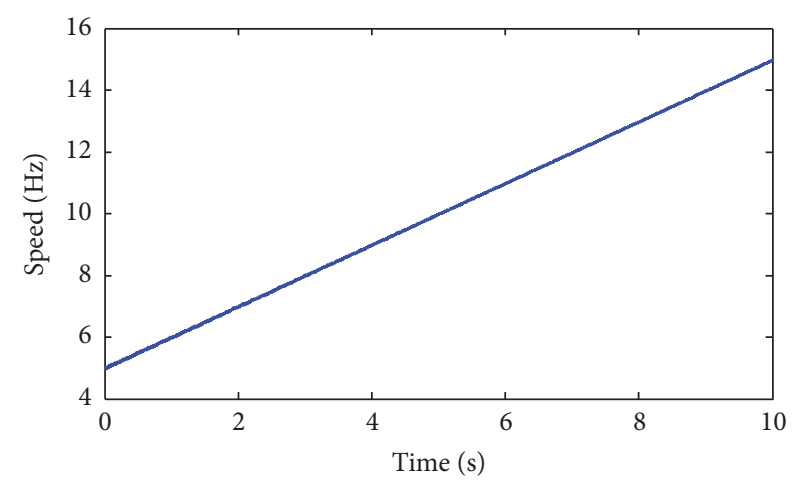

(a)

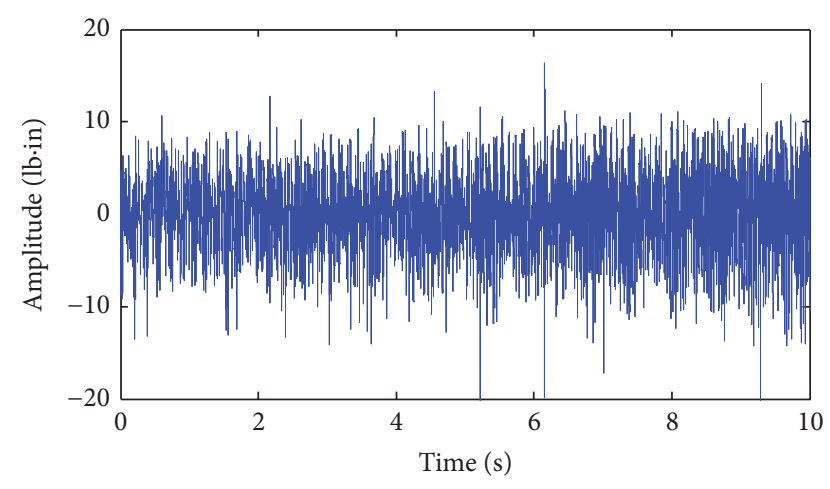

(b)

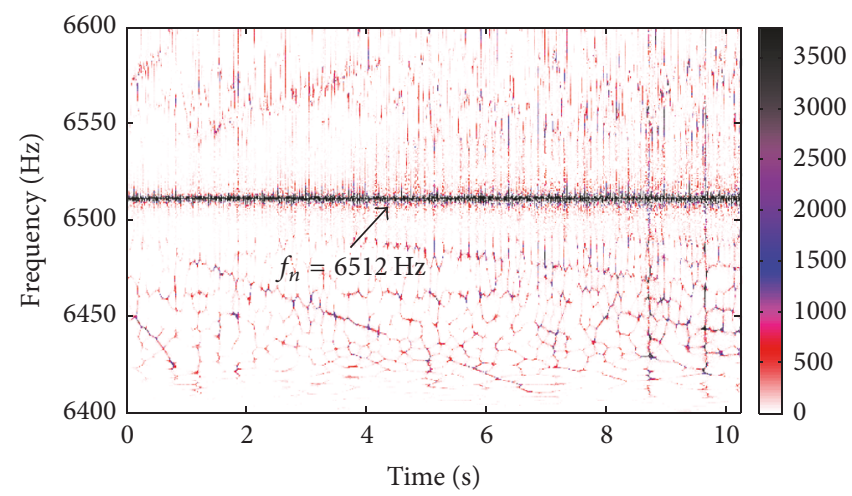

(c)

FIGURE 14: Resonance frequency identification: (a) drive motor speed, (b) detrended torsional vibration signal, and (c) reassigned Morlet scalogram.

of the ring gear fault frequency $1 / 3 n f_{r}$. The presence of $1 / 3$ harmonics of the ring gear fault frequency is reasonable, because of nonidentical planet gears. In Fourier spectrum, and amplitude and frequency demodulation spectra, all the peaks related to the ring gear fault frequency are higher than the baseline signal. This feature implies the ring gear fault.

The above analyses illustrate the effectiveness of our proposed approach in diagnosing planetary gearbox faults via torsional vibration signal analysis in resonance region.

\section{Conclusions}

Torsional vibration signals are free from the additional amplitude modulation effect due to time-varying vibration transmission paths, and they have simpler spectral structure than translational vibration signals, being able to reflect gear fault more effectively. Localized gear faults generate impulses in load torque, thus exciting torsional resonance vibrations and leading to modulation effect on resonance vibration. Therefore, torsional vibration in resonance region can be modelled as an AM-FM process. Its Fourier spectrum, and amplitude and frequency demodulated spectra are derived explicitly. In Fourier spectrum, the sideband around resonance frequency has a spacing equal to gear fault frequency. In both amplitude and frequency demodulated spectra, peaks directly link to gear fault frequency harmonics. For fault diagnosis based on above theoretical derivations, a torsional resonance frequency identification method via time-frequency analysis of torsional vibration signals during variable speed process is proposed, by exploiting the independence nature of resonance on running speed. The resonance frequency can be recognized as the frequency corresponding to a straight line parallel to the time axis on the time-frequency plane. Given the resonance frequency, gear fault can be diagnosed by sideband analysis, and amplitude and frequency demodulation analysis of the sensitive component in resonance region. The theoretical derivations and proposed approach are illustrated by a numerical simulation and are validated with both dynamics modelling data of a planetary gear set and lab experimental signals of a planetary gearbox.

\section{Conflicts of Interest}

The authors declare that there are no conflicts of interest regarding the publication of this paper.

\section{Acknowledgments}

This work is supported by National Natural Science Foundation of China (51475038). 


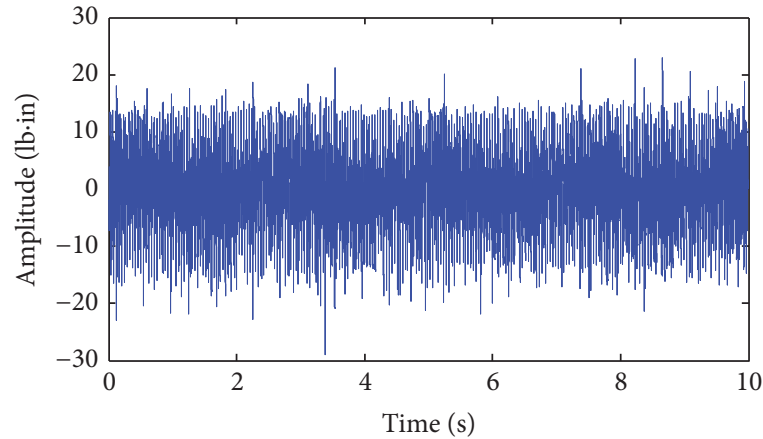

(a)

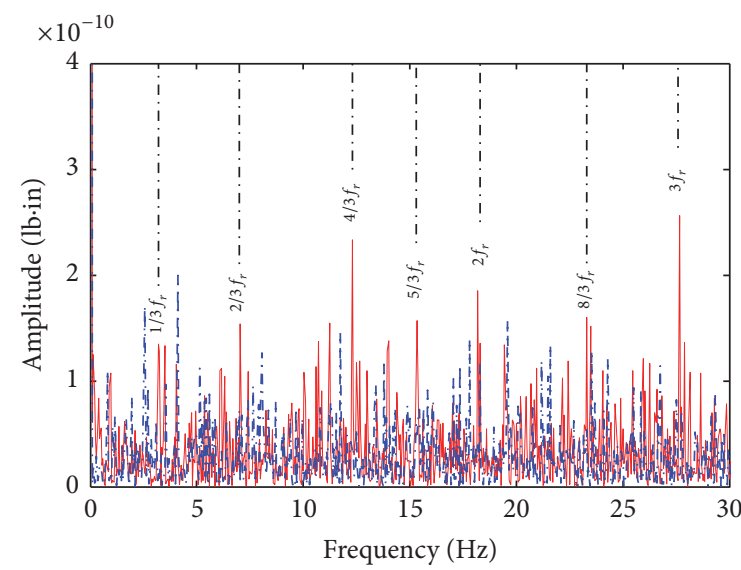

__ Ring gear

-.. Baseline

(c)

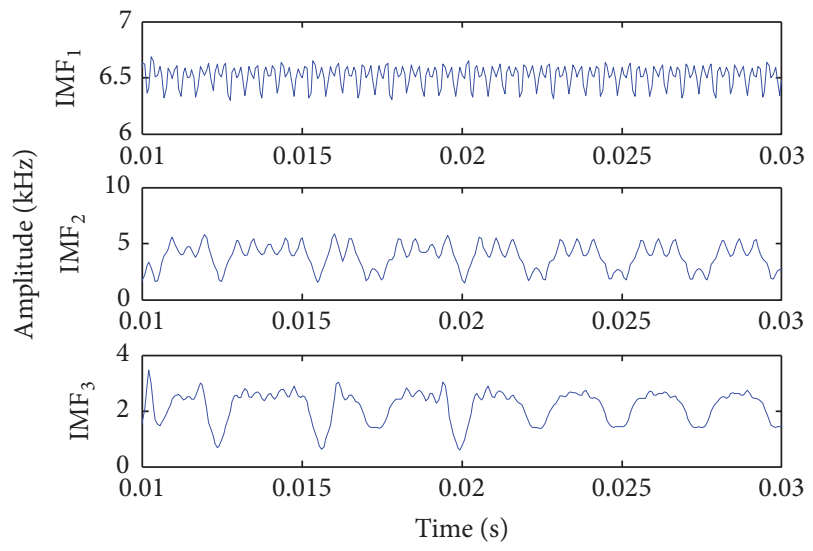

(e)

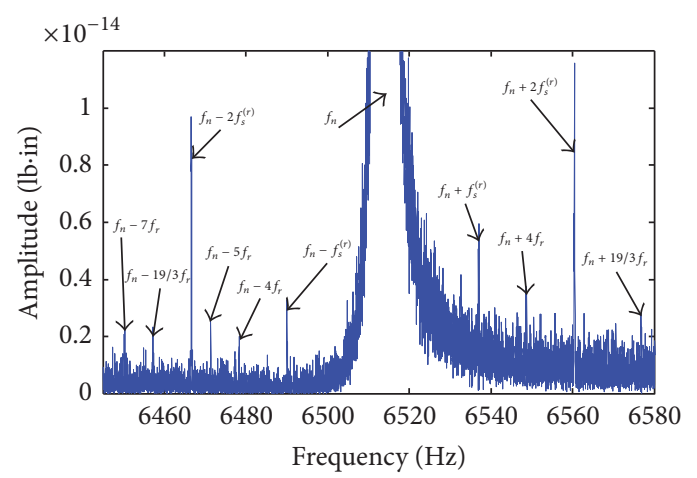

(b)
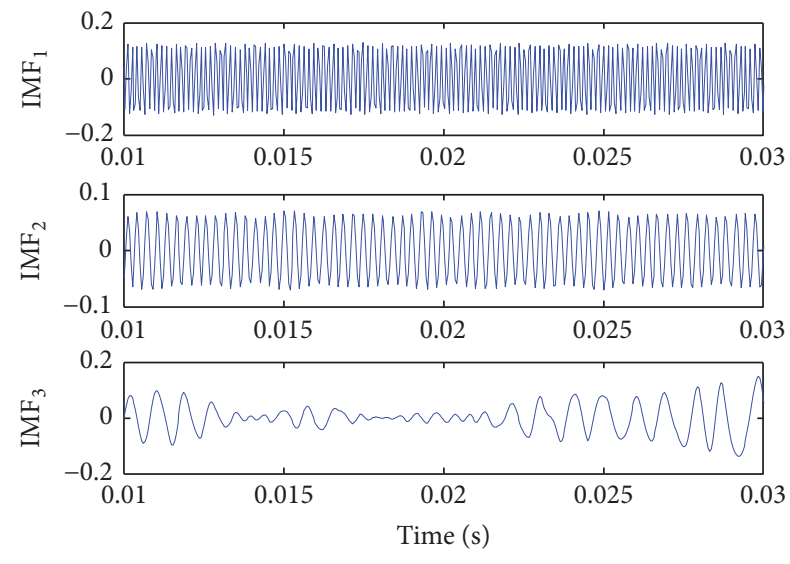

(d)

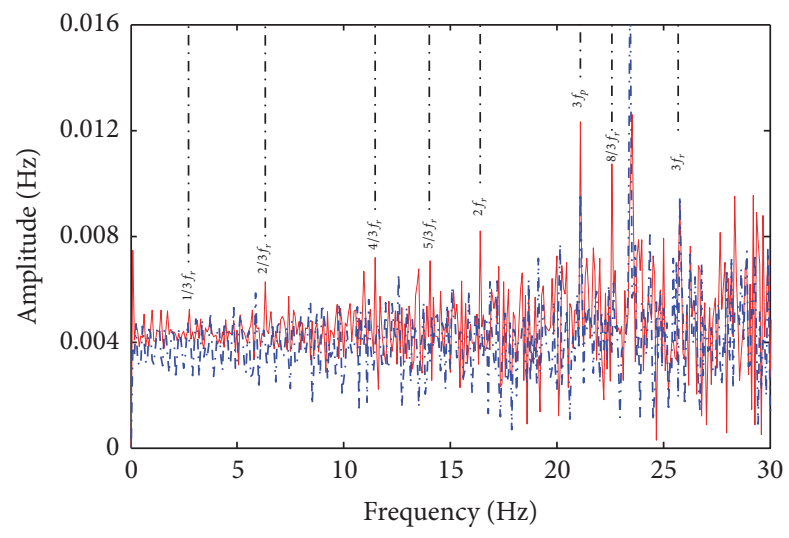

- Ring gear

-..- Baseline

(f)

FIGURE 15: Ring gear fault signal analysis: (a) detrended torsional vibration signal, (b) Fourier spectrum, (c) envelop spectrum, (d) IMFs, (e) instantaneous frequencies of IMFs, and (f) Fourier spectrum of instantaneous frequency.

\section{References}

[1] P. D. Samuel and D. J. Pines, "A review of vibration-based techniques for helicopter transmission diagnostics," Journal of Sound and Vibration, vol. 282, no. 1-2, pp. 475-508, 2005.
[2] Y. G. Lei, J. Lin, M. J. Zuo, and Z. J. He, "Condition monitoring and fault diagnosis of planetary gearboxes: a review," Measurement, vol. 48, no. 2, pp. 292-305, 2014.

[3] P. D. McFadden, "A technique for calculating the time domain averages of the vibration of the individual planet gears and 
the sun gear in an epicyclic gearbox," Journal of Sound and Vibration, vol. 144, no. 1, pp. 163-172, 1991.

[4] P. D. McFadden, "Window functions for the calculation of the time domain averages of the vibration of the individual planet gears and sun gear in an epicyclic gearbox," Journal of Vibration and Acoustics, vol. 116, no. 2, pp. 179-187, 1994.

[5] P. D. Samuel and D. J. Pines, "Vibration separation methodology for planetary gear health monitoring," in Proceedings of the Proceeding of SPIE, vol. 3985, pp. 250-260, March 2000.

[6] X. Liang, M. J. Zuo, and L. Liu, "A windowing and mapping strategy for gear tooth fault detection of a planetary gearbox," Mechanical Systems and Signal Processing, vol. 80, pp. 445-459, 2016.

[7] T. Barszcz and R. B. Randall, "Application of spectral kurtosis for detection of a tooth crack in the planetary gear of a wind turbine," Mechanical Systems and Signal Processing, vol. 23, no. 4, pp. 1352-1365, 2009.

[8] Y. G. Lei, D. Han, J. Lin, and Z. J. He, "Planetary gearbox fault diagnosis using an adaptive stochastic resonance method," Mechanical Systems and Signal Processing, vol. 38, no. 1, pp. 113124, 2013.

[9] J. Yoon, D. He, B. Van Hecke, T. J. Nostrand, J. Zhu, and E. Bechhoefer, "Vibration-based wind turbine planetary gearbox fault diagnosis using spectral averaging," Wind Energy, vol. 19, no. 9, pp. 1733-1747, 2016.

[10] P. D. McFadden and J. D. Smith, "An explanation for the asymmetry of the modulation sidebands about the tooth meshing frequency in epicyclic gear vibration," Proceedings of the Institution of Mechanical Engineers, Part C: Journal of Mechanical Engineering Science, vol. 199, no. 1, pp. 65-70, 1985.

[11] J. McNames, "Fourier series analysis of epicyclic gearbox vibration," Journal of Vibration and Acoustics, vol. 124, no. 1, pp. 150152, 2002.

[12] M. Mosher, "Understanding vibration spectra of planetary gear systems for fault detection," in Proceedings of ASME Design Engineering Technical Conferences, pp. 1-8, 2003.

[13] Z. Feng and M. J. Zuo, "Fault diagnosis of planetary gearboxes via torsional vibration signal analysis," Mechanical Systems and Signal Processing, vol. 36, no. 2, pp. 401-421, 2013.

[14] M. Inalpolat and A. Kahraman, "A theoretical and experimental investigation of modulation sidebands of planetary gear sets," Journal of Sound and Vibration, vol. 323, no. 3-5, pp. 677-696, 2009.

[15] M. Inalpolat and A. Kahraman, "A dynamic model to predict modulation sidebands of a planetary gear set having manufacturing errors," Journal of Sound and Vibration, vol. 329, no. 4, pp. 371-393, 2010.

[16] X. H. Liang, M. J. Zuo, and M. R. Hoseini, "Vibration signal modeling of a planetary gear set for tooth crack detection," Engineering Failure Analysis, vol. 48, pp. 185-200, 2015.

[17] M. Abramowitz and I. A. Stegun, Handbook of Mathematical Functions with Formulas, Graphs, and Mathematical Tables, Dover Publications, New York, NY, USA, 9th edition, 1972.

[18] N. E. Huang, Z. Shen, S. R. Long et al., "The empirical mode decomposition and the Hilbert spectrum for nonlinear and non-stationary time series analysis," Proceedings of the Royal Society A, vol. 454, no. 1971, pp. 903-995, 1998.

[19] Z. H. Wu and N. E. Huang, "Ensemble empirical mode decomposition: a noise-assisted data analysis method," Advances in Adaptive Data Analysis (AADA), vol. 1, no. 1, pp. 1-41, 2009.
[20] Z. Feng and M. J. Zuo, "Vibration signal models for fault diagnosis of planetary gearboxes," Journal of Sound and Vibration, vol. 331, no. 22, pp. 4919-4939, 2012.

[21] Z. P. Feng, M. Liang, Y. Zhang, and S. M. Hou, "Fault diagnosis for wind turbine planetary gearboxes via demodulation analysis based on ensemble empirical mode decomposition and energy separation," Journal of Renewable Energy, vol. 47, pp. 112-126, 2012. 


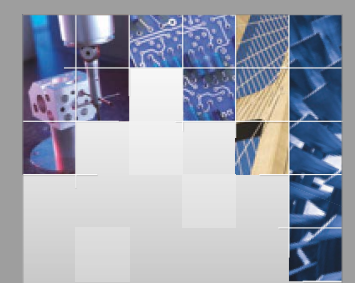

\section{Enfincering}
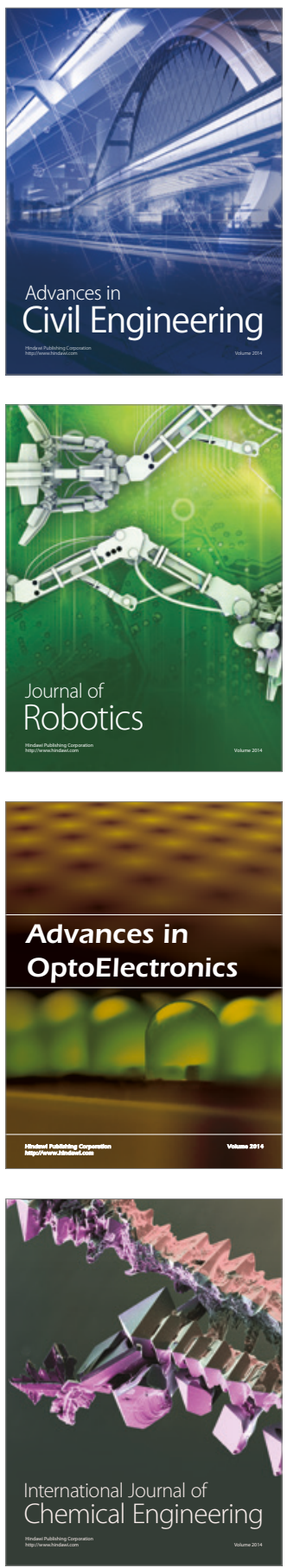

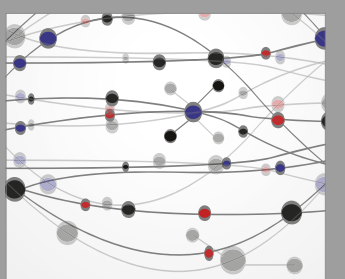

The Scientific World Journal

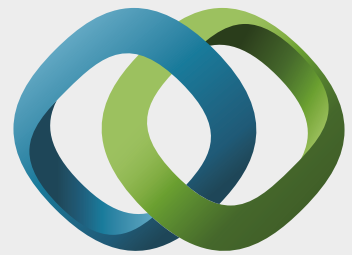

\section{Hindawi}

Submit your manuscripts at

https://www.hindawi.com
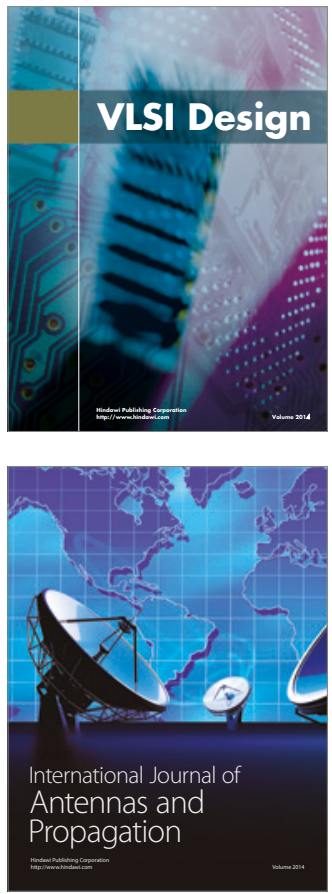

\section{Rotating}

Machinery
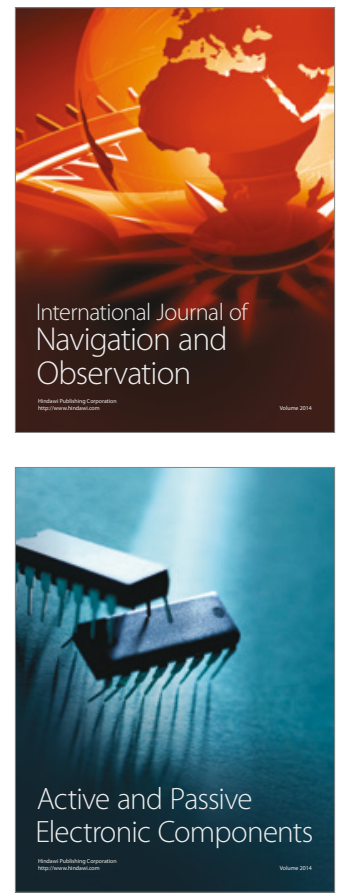
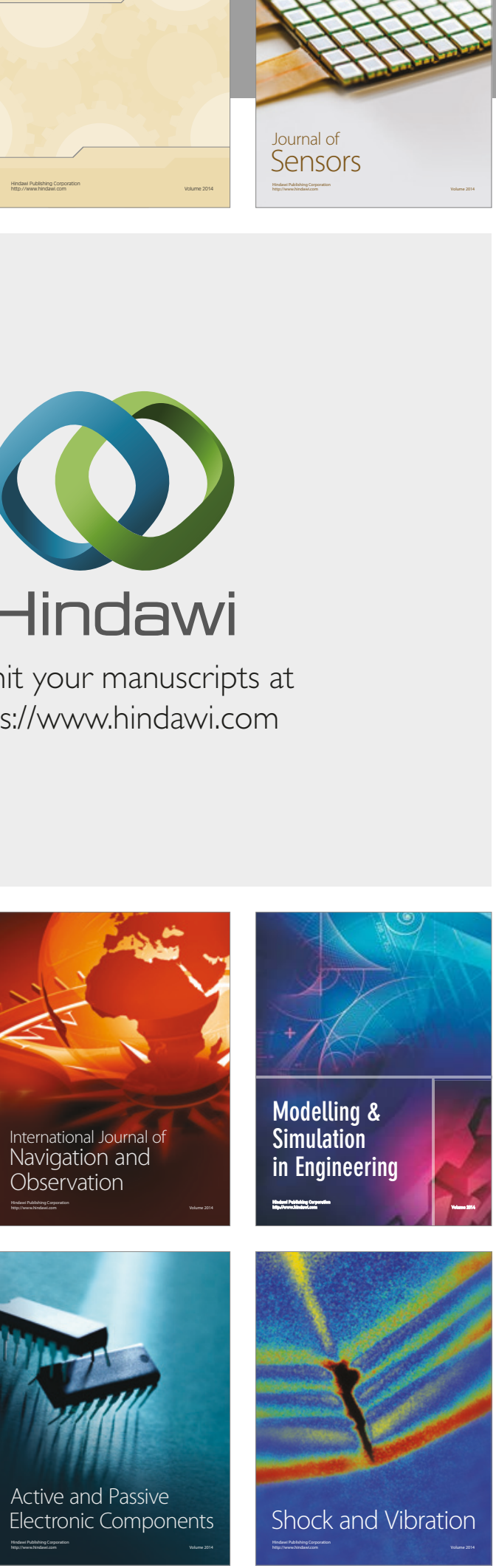
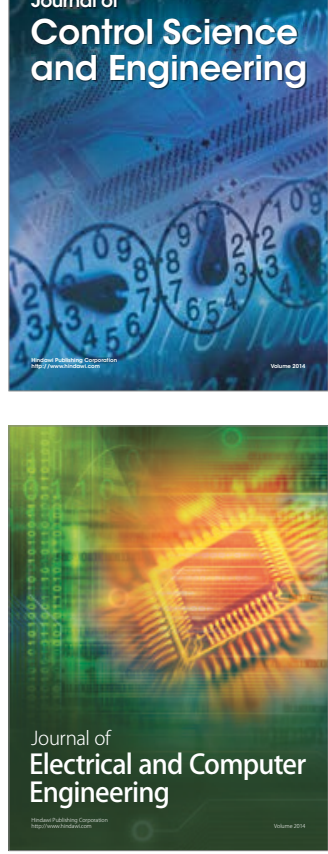

Distributed

Journal of

Control Science

and Engineering
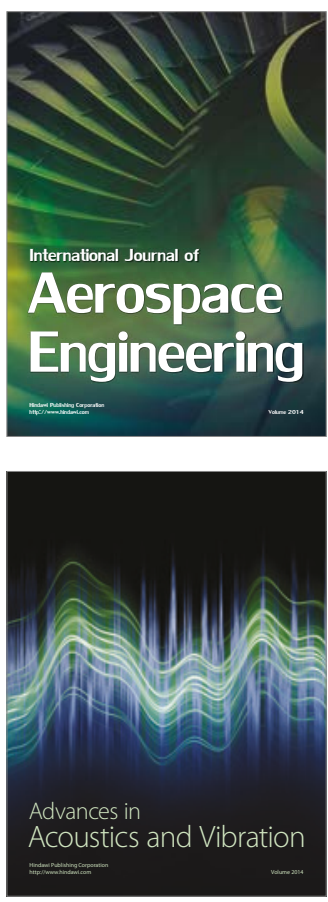

Sensor Networks 